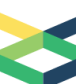

\title{
Two-Dimensional Stress Analysis of Isotropic Deep Beams Using the Finite Difference Method
}

\section{Valentin Fogang ( $\nabla$ valentinfogang@yahoo.de )}

Société BUNS sarl https://orcid.org/0000-0003-1256-9862

\section{Research Article}

Keywords: Finite difference method, additional nodes, Airy stress function, displacement potential function, deep beam of varying thickness, layered beam, deep beam having openings, skew edge, buckling analysis

Posted Date: January 26th, 2022

DOI: https://doi.org/10.21203/rs.3.rs-1298469/v1

License: (c) (1) This work is licensed under a Creative Commons Attribution 4.0 International License. Read Full License 
Article

\title{
Two-Dimensional Stress Analysis of Isotropic Deep Beams Using the Finite Difference Method
}

\author{
Valentin Fogang \\ Civil Engineer, c/o BUNS Sarl, P.O Box 1130, Yaounde, Cameroon; valentinfogang@yahoo.de \\ ORCID iD https://orcid.org/0000-0003-1256-9862
}

\begin{abstract}
This paper presents an approach to the two-dimensional analysis of elastic isotropic deep beams using the finite difference method (FDM). Deep beams are subjected to in-plane loading and present a shear span to height ratio less than 2.50; consequently, Euler-Bernoulli beam theory and Timoshenko beam theory do not apply. Deep beams analysis is generally conducted using numerical methods such as the finite element method and to a lesser extent the FDM; the strut-and-tie model and the stress field method are also widely utilized. Analytical approaches usually make use of the Airy stress function, where stresses are formulated in terms of the stress function; however, the exact solution of this function satisfying all of the boundary conditions can hardly be found, even for simple cases. In this paper, deep beams were analyzed using the FDM. The FDM is an approximate method for solving problems described with differential equations. The FDM does not involve solving differential equations; equations are formulated with values at selected nodes of the structure. Therefore, the deep beam was discretized with a two-dimensional grid, and additional nodes were introduced at the boundaries and at positions of discontinuity (openings, brutal change of material properties, non-uniform grid spacing), the number of additional nodes corresponding to the number of boundary conditions at the node of interest. The introduction of additional nodes allowed us to apply the governing equations at boundary nodes and to satisfy the boundary and continuity conditions. An Airy stress function approach and a displacement potential function approach were considered in this study whereby strong formulations of equations (equilibrium, kinematic, and constitutive) were set. Stress and stability analyses were carried out with this model; furthermore, deep beams of varying stiffness, layered beams, and beams having openings were analyzed. For slender beams, the results obtained with the Airy stress function approach showed good agreement with those of Euler-Bernoulli beam theory, and for deep beams, the shapes of stress distributions were in good agreement with a proper understanding of the behavior of structures. On the other hand, the displacement potential function approach delivered unsatisfactory results, probably due to the use of an inefficient equation solver; a more powerful tool will be needed in future research for this purpose.
\end{abstract}

Keywords: Finite difference method; additional nodes; Airy stress function; displacement potential function; deep beam of varying thickness; layered beam; deep beam having openings; skew edge; buckling analysis 


\section{Stress Analysis of Isotropic Deep Beams Using the Finite Difference Method}

\section{Introduction}

This paper describes the application of Fogang's model [1] based on the finite difference method (FDM), used for the Euler-Bernoulli beam, to the isotropic deep beam. Deep beams are essentially subjected to in-plane loading and present a shear span to height ratio is less than 2.50; consequently, Euler-Bernoulli beam theory and Timoshenko beam theory do not apply. The analytical approach to deep beam analysis is based on the Airy stress function method; this method developed in 1862 by G.B. Airy [2] consists of introducing a new function, the Airy stress function. The stresses were formulated in terms of this new function and a new differential equation, a biharmonic equation, was obtained. The problem of determining the stresses was then reduced to that of finding the stress function solution of the biharmonic equation which satisfies the boundary conditions. Neau [3] developed a scheme for applying doubly infinite power series to the Airy stress function for isotropic bodies; problems in which boundary stresses can be described by means of power series are solvable by this method. Jayne et al. [4] solved the characteristic fourth-order partial differential equation for two-dimensional elastic anisotropic and orthotropic materials, using a doubly infinite power series. Ahmed et al. [5] used an ideal mathematical model, based on a displacement potential function, to formulate the problem; displacements and stresses were formulated in terms of this potential function and a new differential equation, a biharmonic equation, was obtained.

However, the exact solution of the differential equation satisfying the boundary conditions can hardly be found, even for simple cases. Numerical methods permit therefore to overcome solving the differential equations. Ismail et al. [6] performed a series of nonlinear finite element to evaluate the different design approaches available in the literature for design of reinforced concrete deep beam with large opening; three finite element models were developed and analyzed using the computer software ATENA.Vilar et al. [7] proposed a numerical solution to deep beams using the layerwise displacement theory; a finite element solution for deep beams based on a layerwise displacement field considering the full stress/strain tensors was provided. Sri Harsha et al. [8] gave the analytical investigation of reinforced concrete deep beams reinforced with horizontal and vertical web reinforcement; a formula using nonlinear finite element method by ABAQUS was proposed to define the shear strength of deep beams.

For the design of structural concrete, Schlaich et al. [9] proposed a generalization of the truss analogy in order to apply it in the form of strut-and tie-models to every part of any structure; it was described how strut-and tie-models, which condense all stresses in compression and tension members and join them by nodes, can be developed by following the path of the forces throughout a structure. Liu et al. [10] proposed a model for deep beams with rectangular openings that stems from a two-parameter kinematic theory for solid beams; the model was established based on an analysis of the shear behavior and failure modes of test specimens using nonlinear finite element and strut-and-tie models. Silveira et al. [11] proposed a solution based on the stress field method for the analysis, design, and detailing of deep beams. The stress field method, an alternative method to strut-and tie-method for concrete structures subjected to discontinuities, consists of finding the stresses acting in discrete area elements whereby non-linear elastic-plastic stress fields are used. De Mello et al. [12] presented the stringer-panel method, an alternative procedure to strut-and-tie method; the structure is divided on two distinct elements: stringers, which absorb normal forces, and panels, which absorb shear forces by membrane action. Then the overall structure behavior is investigated by means of non-linear analysis. 


\section{Stress Analysis of Isotropic Deep Beams Using the Finite Difference Method}

In the classical analysis using the FDM, points outside the deep beam are not considered; the boundary conditions are applied at the beam's boundaries and not the governing equations. Consequently, the non-application of the governing equations at the boundaries have led to inaccurate results, making the FDM less interesting in comparison to other numerical methods such as the finite element method. In this paper, a model based on FDM was presented. This model consisted of formulating the differential equations with finite differences and introducing additional nodes outside the beam and at positions of discontinuity (openings, brutal change of stiffness's, non-uniform grid spacing). The introduction of additional nodes allowed us to apply the governing equations at the boundaries and to satisfy the boundary and continuity conditions. An Airy [2] stress function approach and a displacement potential function approach of Ahmed et al. [5] were considered whereby strong formulations of equations (equilibrium, kinematic, and constitutive) were set. In the Airy stress function approach stresses were formulated in terms of the Airy stress function whereas in the displacement potential function approach displacements and stresses were formulated in terms of the potential function.

\section{Materials and methods}

\subsection{Governing equations of deep beams}

\subsubsection{Equations of the theory of elasticity}

In this section the equations of the theory of elasticity are recalled. Figure 1 represents a deep beam with the axis convention $(\mathrm{X}, \mathrm{Y})$.
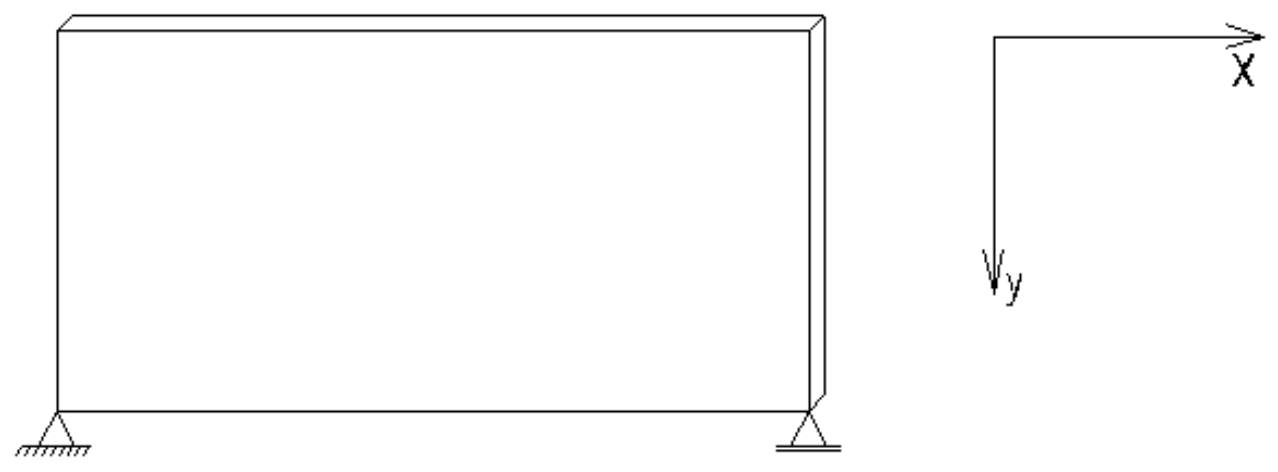

Figure 1 Deep beam with axis convention $\mathrm{X}, \mathrm{Y}$

Displacements in $\mathrm{x}$ - and $\mathrm{y}$ - directions are denoted by $\mathrm{u}(x, y)$ and $\mathrm{v}(x, y)$, respectively. The axial strains $\varepsilon_{\mathrm{xx}}$ and $\varepsilon_{\mathrm{yy}}$ and the shearing strain $\gamma_{\mathrm{xy}}$ are related to the displacements as follows:

$$
\varepsilon_{x x}=\frac{\partial u}{\partial x}, \quad \varepsilon_{y y}=\frac{\partial v}{\partial y}, \quad \gamma_{x y}=\frac{\partial u}{\partial y}+\frac{\partial v}{\partial x}
$$

Combining Equations (1a-c) yields the following compatibility condition between axial strains and shearing strain

$$
\frac{\partial^{2} \gamma_{x y}}{\partial x \partial y}=\frac{\partial^{2} \varepsilon_{x x}}{\partial y^{2}}+\frac{\partial^{2} \varepsilon_{y y}}{\partial x^{2}}
$$


Assuming a plane stress distribution, the relationships between stresses and strains are given by

$\varepsilon_{x x}=\frac{\sigma_{x x}}{E}-v \frac{\sigma_{y y}}{E}, \quad \varepsilon_{y y}=\frac{\sigma_{y y}}{E}-v \frac{\sigma_{x x}}{E}, \quad \gamma_{x y}=\frac{\tau_{x y}}{G}$

where E is the Young's modulus and $v$ is the Poisson's ratio. Combining Equations (1a-c) and (2a-c) yields

$\sigma_{x x}=\frac{E}{1-v^{2}} \times\left(\frac{\partial u}{\partial x}+v \frac{\partial v}{\partial y}\right), \quad \sigma_{y y}=\frac{E}{1-v^{2}} \times\left(\frac{\partial v}{\partial y}+v \frac{\partial u}{\partial x}\right)$,

$\tau_{x y}=G \gamma_{x y}=\frac{E}{2(1+v)} \times\left(\frac{\partial u}{\partial y}+\frac{\partial v}{\partial x}\right)$

The equations of static equilibrium on an infinitesimal beam element of dimensions dx, dy, and thickness $d$ are given by

$$
\frac{\partial \sigma_{x x}}{\partial x}+\frac{\partial \tau_{x y}}{\partial y}+p_{x}=0, \quad \frac{\partial \sigma_{y y}}{\partial y}+\frac{\partial \tau_{x y}}{\partial x}+p_{y}=0
$$

where $p_{\mathrm{x}}$ and $\mathrm{p}_{\mathrm{y}}$ are body forces $\left[\mathrm{kN} / \mathrm{m}^{3}\right]$. Substituting Equations (3a-c) into (4a-b) yields

$$
\begin{aligned}
& 2 \frac{\partial^{2} u}{\partial x^{2}}+(1-v) \frac{\partial^{2} u}{\partial y^{2}}+(1+v) \frac{\partial^{2} v}{\partial x \partial y}+2 \frac{p_{x}}{D}=0, \\
& 2 \frac{\partial^{2} v}{\partial y^{2}}+(1-v) \frac{\partial^{2} v}{\partial x^{2}}+(1+v) \frac{\partial^{2} u}{\partial x \partial y}+2 \frac{p_{y}}{D}=0,
\end{aligned}
$$

where $\mathrm{D}$ is the axial rigidity of the deep beam. The solutions considered in the present study and presented thereafter involve a two displacement function, an Airy stress function, and a displacement potential function.

\subsubsection{Two displacement function}

The two displacement function approach is governed by Equations (5a-b) which are applied at any node of the structure whereby the displacements are the unknowns. The geometric boundary conditions are directly formulated whereas stress related boundary conditions are satisfied using Equations (3a-c).

\subsubsection{Airy stress function}

G. B. Airy [2] introduced a function $\phi(x, y)$, defined in terms of stress components, to formulate the analysis. In the absence of body forces on the structure, this function was related to the axial/shearing stresses as follows

$$
\sigma_{x x}=\frac{\partial^{2} \phi}{\partial y^{2}}, \quad \sigma_{y y}=\frac{\partial^{2} \phi}{\partial x^{2}}, \quad \tau_{x y}=-\frac{\partial^{2} \phi}{\partial x \partial y}
$$




\section{Stress Analysis of Isotropic Deep Beams Using the Finite Difference Method}

It is noted that Equations (6a-c) satisfy the equilibrium equations (4a-b). Substituting Equations (6a-c) into (2a-c), and the result into (1d) yields the following condition to be satisfied by the stress function $\phi(x, y)$

$$
\frac{\partial^{4} \phi}{\partial x^{4}}+2 \frac{\partial^{4} \phi}{\partial x^{2} \partial y^{2}}+\frac{\partial^{4} \phi}{\partial y^{4}}=0
$$

Combining Equations (1a-b), (2a-b), and (6a-b) yields the following relationship between the Airy stress function (ASF) and the rates of change of displacements

$$
\begin{aligned}
& \varepsilon_{x x}=\frac{\partial u}{\partial x}=\frac{1}{E}\left(\frac{\partial^{2} \phi}{\partial y^{2}}-v \frac{\partial^{2} \phi}{\partial x^{2}}\right) \\
& \varepsilon_{y y}=\frac{\partial v}{\partial y}=\frac{1}{E}\left(\frac{\partial^{2} \phi}{\partial x^{2}}-v \frac{\partial^{2} \phi}{\partial y^{2}}\right)
\end{aligned}
$$

The analysis is then reduced of determining one single function, the ASF. However, satisfying the geometric boundary conditions is not easy with this solution since the displacements are not specified in terms of the stress function. If constant body forces $\mathrm{p}_{\mathrm{x}}$ and $\mathrm{p}_{\mathrm{y}}$ are applied, Equations (6a-c) can be modified using Säckel [13] as follows

$$
\sigma_{x x}=\frac{\partial^{2} \phi}{\partial y^{2}}, \quad \sigma_{y y}=\frac{\partial^{2} \phi}{\partial x^{2}}, \quad \tau_{x y}=-\frac{\partial^{2} \phi}{\partial x \partial y}-y p_{x}-x p_{y}
$$

\subsubsection{Displacement potential function}

In case no body forces were applied on the structure, Ahmed et al. [5] introduced a displacement potential function (DPF) $\psi(\mathrm{x}, \mathrm{y})$, defined in terms of displacement components. However, the Cartesian coordinates $\mathrm{x}, \mathrm{y}$ and the displacements $\mathrm{u}(\mathrm{x}, \mathrm{y})$ and $\mathrm{v}(\mathrm{x}, \mathrm{y})$ of Ahmed et al. [5] have to be inverted in order to be consistent with the axis convention of the present study. Therefore, the displacements are related to the potential function are as follows

$$
u(x, y)=-\frac{1}{1+v}\left[(1-v) \frac{\partial^{2} \psi}{\partial x^{2}}+2 \frac{\partial^{2} \psi}{\partial y^{2}}\right], \quad v(x, y)=\frac{\partial^{2} \psi}{\partial x \partial y}
$$

Equations (9a-b) satisfy automatically the equilibrium equation, Equation (5b). Equation (5a) implies that the following condition be satisfied by $\psi(\mathrm{x}, \mathrm{y})$

$$
\frac{\partial^{4} \psi}{\partial x^{4}}+2 \frac{\partial^{4} \psi}{\partial x^{2} \partial y^{2}}+\frac{\partial^{4} \psi}{\partial y^{4}}=0
$$

Substituting Equations (9a-b) into (3a-c) yields the axial/shearing stresses as follows

$$
\begin{array}{ll}
\sigma_{x x}=-\frac{E}{(1+v)^{2}} \times\left[\frac{\partial^{3} \psi}{\partial x^{3}}+(2+v) \frac{\partial^{3} \psi}{\partial x \partial y^{2}}\right], & \sigma_{y y}=\frac{E}{(1+v)^{2}} \times\left(\frac{\partial^{3} \psi}{\partial x \partial y^{2}}-v \frac{\partial^{3} \psi}{\partial x^{3}}\right) \\
\tau_{x y}=\frac{E}{(1+v)^{2}} \times\left(v \frac{\partial^{3} \psi}{\partial x^{2} \partial y}-\frac{\partial^{3} \psi}{\partial y^{3}}\right) &
\end{array}
$$


Similarly to the solution using the ASF, the analysis is reduced of determining one single function, the DPF; interestingly, the satisfaction of boundary conditions is facilitated in this solution, since displacements and stresses are specified in terms of the potential function.

If constant body forces $p_{x}$ and $p_{y}$ are applied, Equations $(9 a, b)$ of Ahmed et al. [5] can be modified as follows

$$
u(x, y)=-\frac{1}{1+v}\left[(1-v) \frac{\partial^{2} \psi}{\partial x^{2}}+2 \frac{\partial^{2} \psi}{\partial y^{2}}\right]-\frac{p_{x}}{2 D} x^{2}, \quad v(x, y)=\frac{\partial^{2} \psi}{\partial x \partial y}-\frac{p_{y}}{2 D} y^{2}
$$

The expression of the shearing stress is unchanged whereas that of axial stresses becomes

$$
\begin{aligned}
& \sigma_{x x}=-\frac{E}{(1+v)^{2}} \times\left[\frac{\partial^{3} \psi}{\partial x^{3}}+(2+v) \frac{\partial^{3} \psi}{\partial x \partial y^{2}}\right]-x p_{x}-v y p_{y}, \\
& \sigma_{y y}=\frac{E}{(1+v)^{2}} \times\left(\frac{\partial^{3} \psi}{\partial x \partial y^{2}}-v \frac{\partial^{3} \psi}{\partial x^{3}}\right)-y p_{y}-v x p_{x}
\end{aligned}
$$

\subsection{Finite difference approximations for a deep beam}

\subsubsection{Fundamentals of finite difference approximations}

The two displacement function approach will not be considered further for the stress analysis since two equations (the governing equations (5a-b)) have to be set at any node, whereas only one equation is set at any node for the solutions using the Airy stress function (ASF) and the solution using the potential function. First, Equation (7) is the governing equation for the ASF approach. This equation has fourth order derivatives; consequently, the stress function $\phi(x, y)$ is approximated around the node of interest $\mathbf{i}$ as a fourth degree polynomial in each direction. The unknown at any node being the value $\phi_{i}$ of the stress function, the corresponding finite difference approximation (FDA) is denoted by ASF-FDA. Second, Equation (9c) is the governing equation in case the DPF $\psi(\mathrm{x}, \mathrm{y})$ is considered. The unknown at any node being the value $\psi_{\mathrm{i}}$ of the potential function, the corresponding FDA is denoted by DPF-FDA

\subsubsection{Airy stress function finite difference approximation}

Given the grid spacings $\Delta \mathbf{x}=\mathbf{h}$ and $\Delta \mathbf{y}=\lambda \mathbf{h}$ in $\mathrm{x}$ - and $\mathrm{y}$-direction, respectively. The stress function $\phi(\mathrm{x}, \mathrm{y})$ is approximated around the node of interest $i$ as a fourth order polynomial in each direction; however, for simplification purpose, the first and second partial derivatives in x-direction and the mixed partial derivative $\partial^{2} \phi / \partial x \partial y$ are expressed using a second order polynomial hypothesis for $\phi(\mathrm{x}, \mathrm{y})$. The FDAs are then given by 


$$
\begin{aligned}
& \left.\frac{\partial^{2} \phi}{\partial x^{2}}\right|_{i-1}=\left.\frac{\partial^{2} \phi}{\partial x^{2}}\right|_{i}=\left.\frac{\partial^{2} \phi}{\partial x^{2}}\right|_{i+1}=\frac{\phi_{i-1}-2 \phi_{i}+\phi_{i+1}}{h^{2}},\left.\quad \frac{\partial^{2} \phi}{\partial x \partial y}\right|_{i}=\frac{1}{4 \lambda h^{2}}\left[\begin{array}{ccc}
1 & 0 & -1 \\
0 & {[0]} & 0 \\
-1 & 0 & 1
\end{array}\right] \times[\phi] \\
& {\left[\left.\left.\left.\frac{\partial \phi}{\partial x}\right|_{i-1} \quad \frac{\partial \phi}{\partial x}\right|_{i} \frac{\partial \phi}{\partial x}\right|_{i+1}\right]^{T}=\frac{1}{2 h}\left[\begin{array}{ccc}
-3 & 4 & -1 \\
-1 & 0 & 1 \\
1 & -4 & 3
\end{array}\right] \times[\phi]}
\end{aligned}
$$

In the stencil notation the factor associated to the node of interest is in brackets. The partial derivatives in y-direction are formulated similarly. The FDAs of the fourth order partial derivatives in x-direction are given by

$$
\left.\frac{\partial^{4} \phi}{\partial x^{4}}\right|_{i-2}=\left.\frac{\partial^{4} \phi}{\partial x^{4}}\right|_{i-1}=\left.\frac{\partial^{4} \phi}{\partial x^{4}}\right|_{i}=\left.\frac{\partial^{4} \phi}{\partial x^{4}}\right|_{i+1}=\left.\frac{\partial^{4} \phi}{\partial x^{4}}\right|_{i+2}=\frac{\phi_{i-2}-4 \phi_{i-1}+6 \phi_{i}-4 \phi_{i+1}+\phi_{i+2}}{h^{4}}
$$

The FDA of the term $\partial^{4} \phi / \partial x^{2} \partial y^{2}$ defined using Equation (10a) is expressed by means of the following stencil

$$
\left.\frac{\partial^{4} \phi}{\partial x^{2} \partial y^{2}}\right|_{k}=\frac{1}{\lambda^{2} h^{4}}\left[\begin{array}{ccc}
1 & -2 & 1 \\
-2 & {[4]} & -2 \\
1 & -2 & 1
\end{array}\right] \times[\phi]
$$

2.2.3

Displacement potential function finite difference approximation

The partial derivatives $\partial^{4} \psi / \partial \mathrm{x}^{4}, \partial^{4} \psi / \partial \mathrm{x}^{2} \partial \mathrm{y}^{2}, \partial^{2} \psi / \partial \mathrm{x}^{2}$ and $\partial^{2} \psi / \partial \mathrm{x} \partial \mathrm{y}$ are expressed similarly to the ASF approach. Using a fourth order polynomial hypothesis for $\psi(\mathrm{x}, \mathrm{y})$, the third derivatives $\partial^{3} \psi / \partial \mathrm{x}^{3}$ at different nodes are expressed in terms of values of $\psi(\mathrm{x}, \mathrm{y})$ as follows

$$
\left[\begin{array}{l}
\psi_{i-2}^{(3)} \\
\psi_{i-1}^{(3)} \\
\psi_{i}^{(3)} \\
\psi_{i+1}^{(3)} \\
\psi_{i+2}^{(3)}
\end{array}\right]=\frac{1}{2 h^{3}}\left[\begin{array}{ccccc}
-5 & 18 & -24 & 14 & -3 \\
-3 & 10 & -12 & 6 & -1 \\
-1 & 2 & 0 & -2 & 1 \\
1 & -6 & 12 & -10 & 3 \\
3 & -14 & 24 & -18 & 5
\end{array}\right] \times\left[\begin{array}{c}
\psi_{i-2} \\
\psi_{i-1} \\
\psi_{i} \\
\psi_{i+1} \\
\psi_{i+2}
\end{array}\right]
$$

Using Equations (10a, c), the FDAs of the partial derivatives $\partial^{3} \psi / \partial \mathrm{x} \partial \mathrm{y}^{2}$ and $\partial^{3} \psi / \partial \mathrm{x}^{2} \partial \mathrm{y}$ are given by

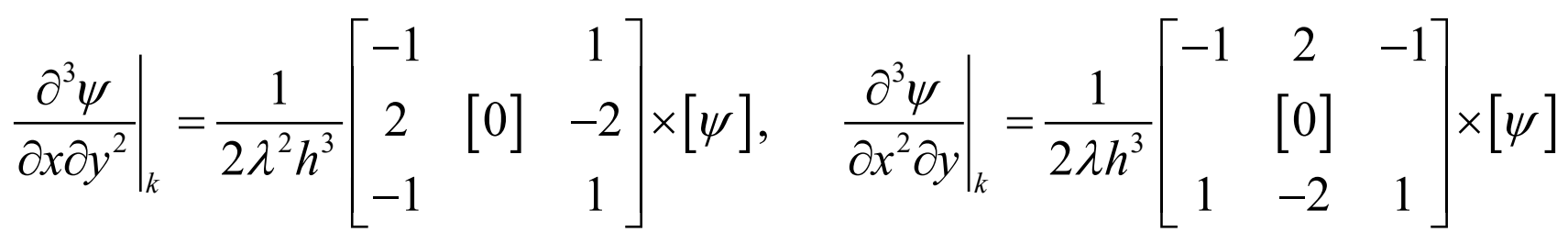




\subsubsection{Finite difference approximations at an interior node}

Figure 2 shows the node distribution of a deep beam having equidistant nodes with spacings $\Delta \mathbf{x}$ and $\Delta \mathbf{y}$ in $\mathrm{x}$ - and $\mathrm{y}$ direction, respectively. The node of interest $\mathbf{k}$ and the surrounding nodes are represented, whereby $\mathrm{n}, \mathrm{s}, \mathrm{e}$, and w stand for the directions north, south, east, and west, respectively, according to the directions in the stencil. The node k may even be at the boundary of the beam, however being not at angles.

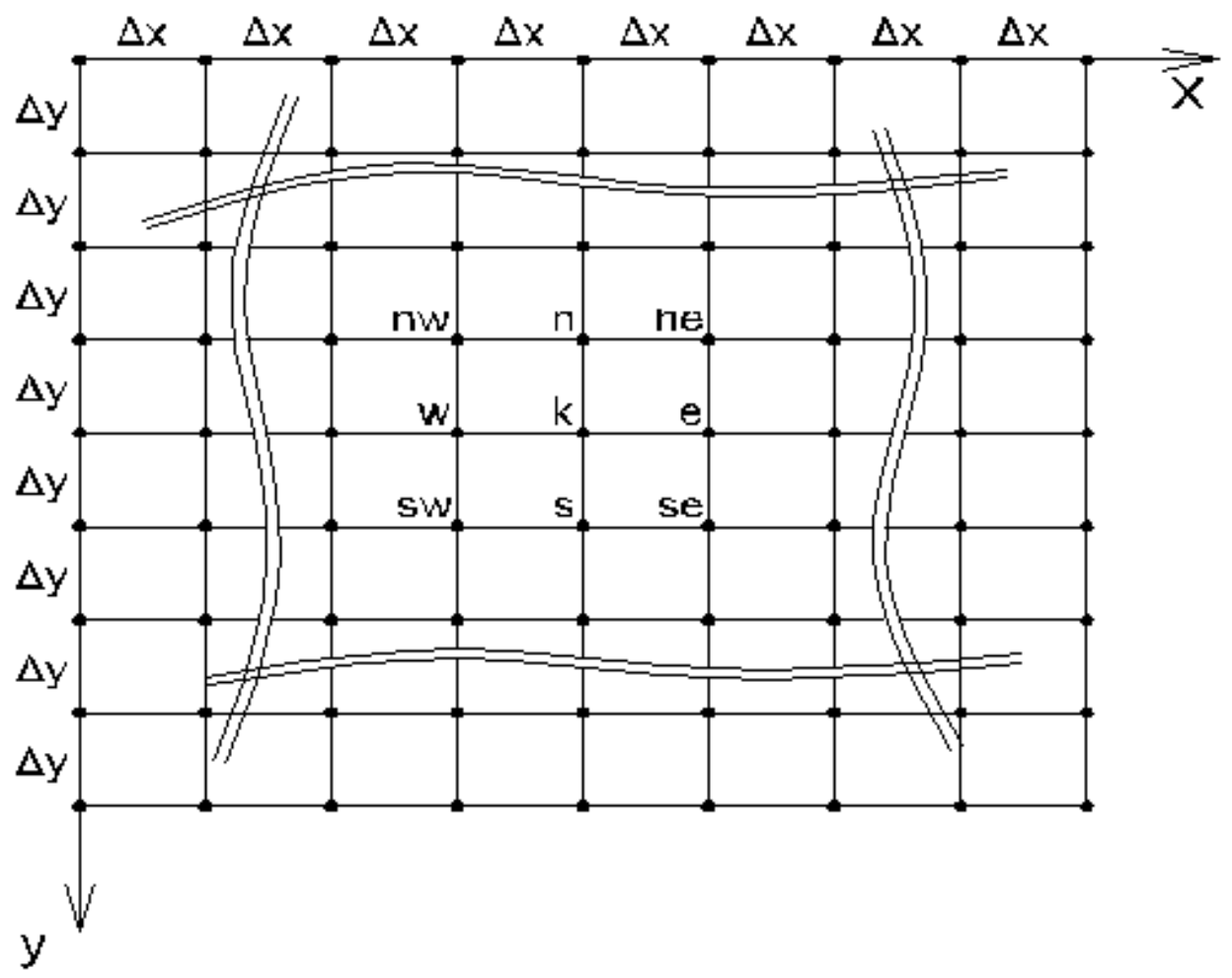

Figure 2 Point of interest $\mathbf{k}$ and its surrounding points

The FDAs for the Airy stress function approach and the displacement potential function approach are determined.

\subsubsection{Airy stress function FDA at an interior node}

Given the grid spacings $\Delta \mathbf{x}=\mathbf{h}$ and $\Delta \mathbf{y}=\lambda \mathbf{h}$. The governing equation (Equation (7)) at a given node can be expressed by means of a stencil using Equations (11) and (11a) as follows

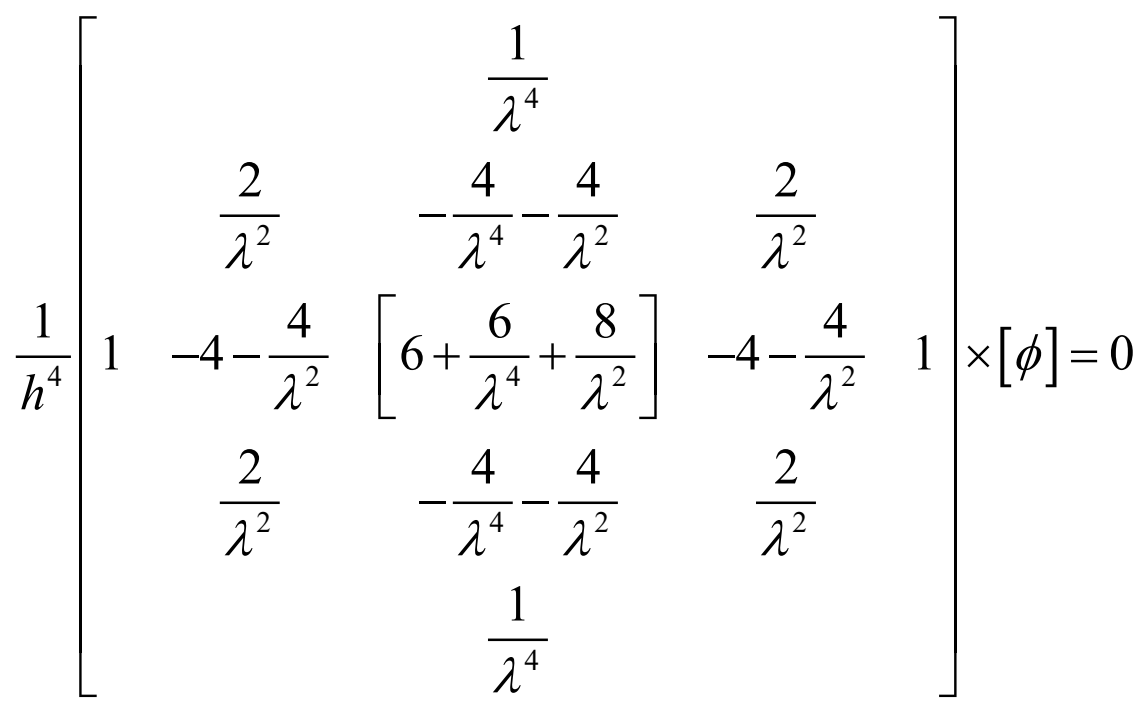


The axial/shearing stresses at a given node are expressed using Equations (6a-c) and (10a-b) as follows;

$\sigma_{x x}=\frac{1}{\lambda^{2} h^{2}}\left[\begin{array}{c}1 \\ 1\end{array}\right] \times[\phi], \quad \sigma_{y y}=\frac{1}{h^{2}}[1 \quad[-2] 1] \times[\phi], \quad \tau_{x y}=-\frac{1}{4 \lambda h^{2}}\left[\begin{array}{cc}1 & -1 \\ -1 & {[0]}\end{array}\right] \times[\phi]$

Introducing following modified displacements,

$$
U^{*}(x, y)=\frac{E h}{2} \times u(x, y), \quad V^{*}(x, y)=\frac{E h}{2} \times v(x, y),
$$

the displacements are calculated using Equations (8a-b), (10a, c) and (15) as follows

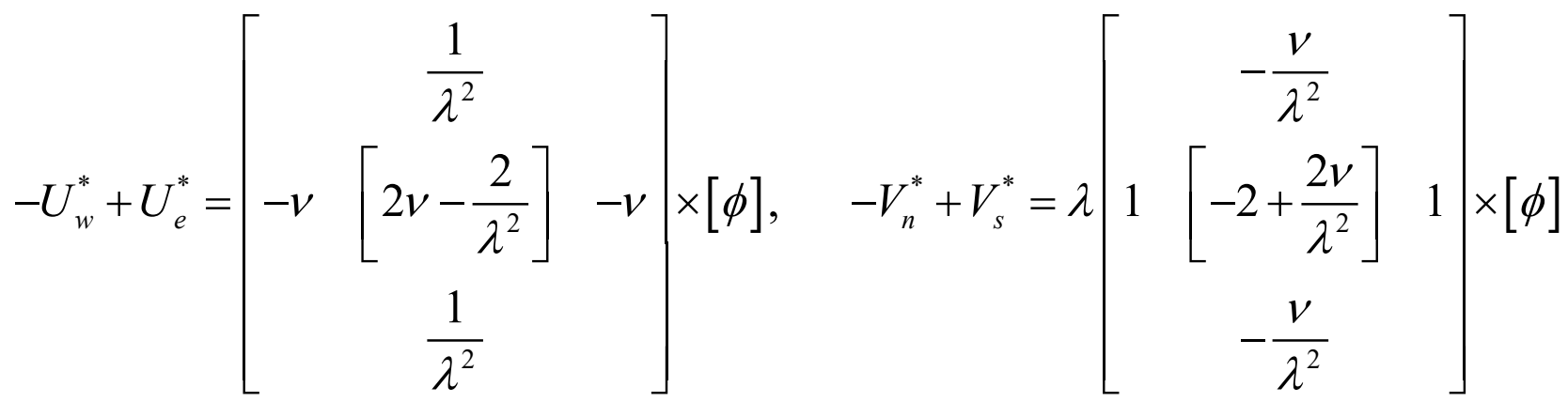

\subsubsection{Displacement potential function FDA at an interior node}

The governing equation (Equation (9c)) can be expressed by means of a stencil using Equations (13). At node i the FDA of $\mathrm{u}(\mathrm{x}, \mathrm{y})$ and $\mathrm{v}(\mathrm{x}, \mathrm{y})$ are formulated using Equations $(9 \mathrm{a}-\mathrm{b})$ and $(10 \mathrm{a}-\mathrm{b})$ as follows

$\left.u_{i}=-\frac{1}{(1+v) h^{2}}\left[\begin{array}{c}\frac{2}{\lambda^{2}} \\ 1-v \quad\left[-2(1-v)-\frac{4}{\lambda^{2}}\right] \\ \frac{2}{\lambda^{2}}\end{array}\right] 1-v\right] \times[\psi], \quad v_{i}=\frac{1}{4 \lambda h^{2}}\left[\begin{array}{ccc}1 & 0 & -1 \\ 0 & {[0]} & 0 \\ -1 & 0 & 1\end{array}\right] \times[\psi]$

The FDA of the axial/shearing stresses are formulated using Equations (9d-f) and (12a-b) as follows

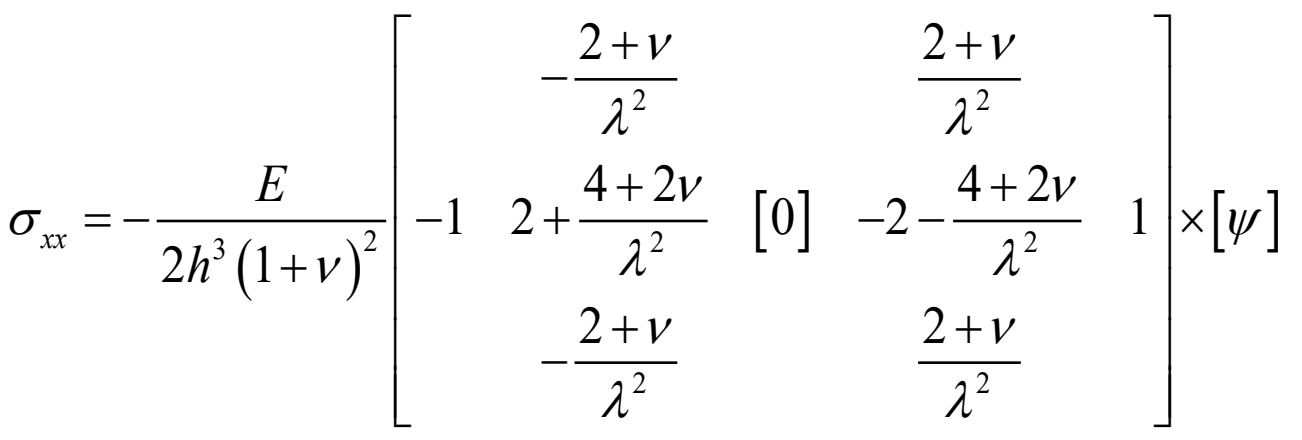


Stress Analysis of Isotropic Deep Beams Using the Finite Difference Method

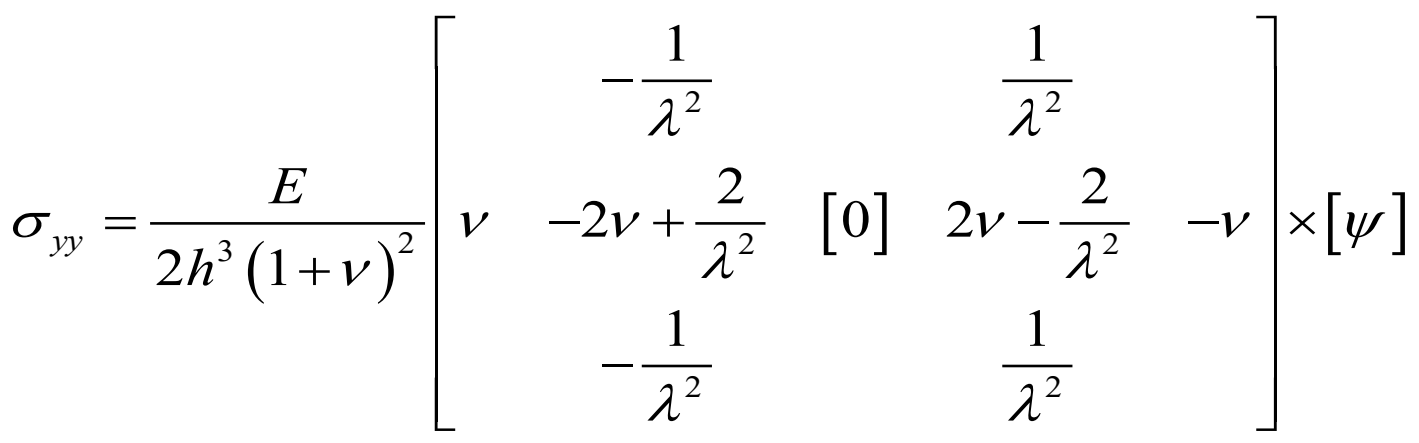

$$
\begin{aligned}
& \tau_{x y}=\frac{E}{2 h^{3}(1+v)^{2}}\left[\begin{array}{ccc}
\frac{1}{\lambda^{3}} & \\
-\frac{v}{\lambda} & -\frac{2}{\lambda^{3}}+\frac{2 v}{\lambda} & -\frac{v}{\lambda} \\
\frac{v}{\lambda} & \frac{2}{\lambda^{3}}-\frac{2 v}{\lambda} & \frac{v}{\lambda} \\
-\frac{1}{\lambda^{3}}
\end{array}\right] \times[\psi]
\end{aligned}
$$

\subsubsection{Finite difference approximation in the vicinity of beam angles}

\subsubsection{Airy stress function in the vicinity of beam angles}

Figure 3 below represents one angle of the beam; regular nodes (k; e; ee; eee; s; se; ss...) and additional nodes (nw; n; ne; w; sw...) are shown. The unknown at each point, regular node or additional node, is the value of the stress function. The number of additional nodes associated to an edge node corresponds to the number of boundary conditions at the node of interest: therefore, three additional nodes are introduced at the angle node $\mathrm{k}$ and two additional nodes at the other edge nodes (e; ee; s; ss ...). 


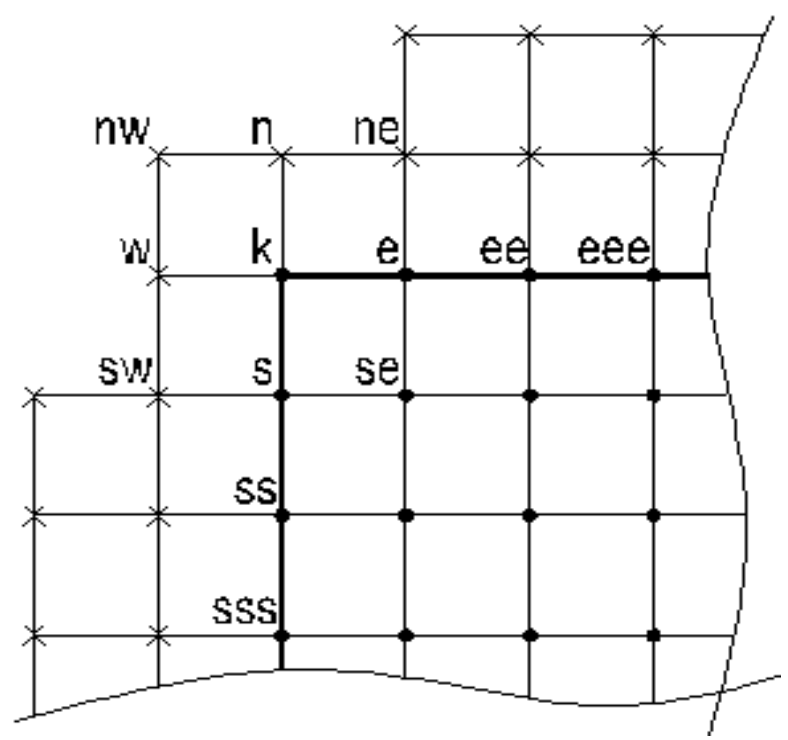

Figure 3 Beam angle with regular nodes $(\bullet)$ and additional nodes $(\times)$

\section{ASF Finite difference approximation at node $k$}

Equations (11) are used for the terms $\partial^{4} \mathrm{~F} / \partial \mathrm{x}^{4}$ and $\partial^{4} \mathrm{~F} / \partial \mathrm{y}^{4}$ while Equation (11a) is used for $\partial^{4} \mathrm{~F} / \partial \mathrm{x}^{2} \partial \mathrm{y}^{2}$. The governing equation (Equation (7)) of the beam at node $\mathrm{k}$ is then described with the following 13-point stencil

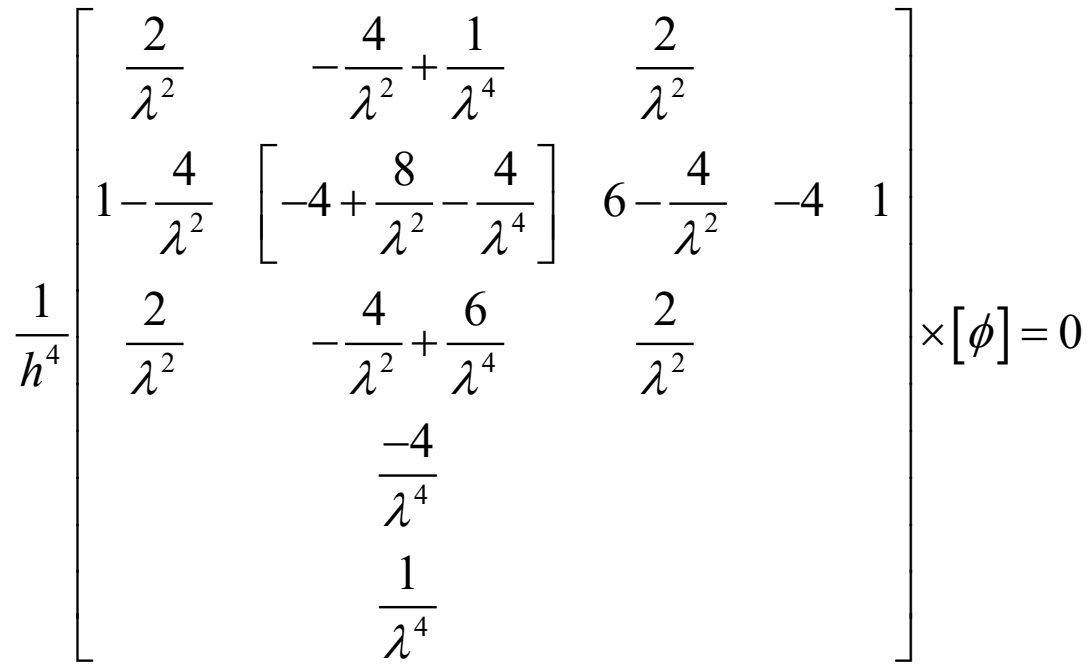

For other angle points of the structure, the governing equations are determined using the same principle, and are presented in Appendix A. The axial/shearing stresses are expressed using Equations (14); therefore the stress related boundary conditions are formulated.

In case of geometric boundary conditions i.e. $\mathrm{u}=0$ at the node $\mathrm{k}$, $\mathrm{u}$ - displacements are introduced at nodes $\mathrm{k}$, e, and ee; for $\mathrm{v}=0$ at the node $\mathrm{k}, \mathrm{v}$ - displacements are introduced at nodes $\mathrm{k}, \mathrm{n}$, and $\mathrm{nn}$, as represented in Figure 4. 


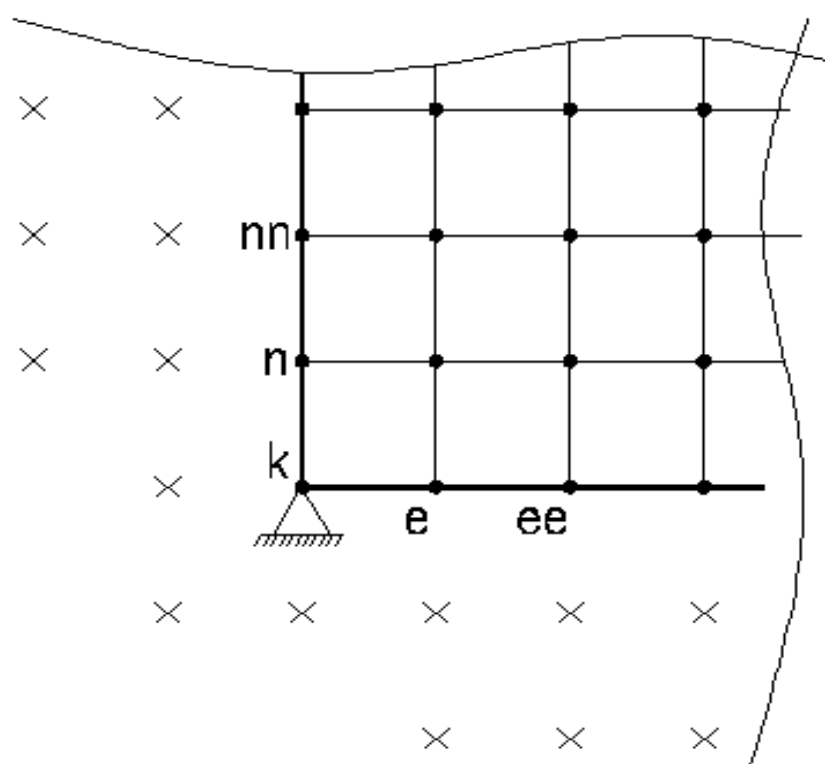

Figure 4 Beam angle with regular nodes $(\bullet)$ and additional nodes $(\times)$

For $\mathrm{u}=0$, Equation (8a) is applied at nodes $\mathrm{k}$, e, and ee using Equations (10c) and (15a) as follows

$$
\begin{aligned}
& -3 U_{k}^{*}+4 U_{e}^{*}-U_{e e}^{*}=\Delta x^{2} \times\left[\frac{\partial^{2} F}{\partial y^{2}}-v \frac{\partial^{2} F}{\partial x^{2}}\right]_{k}, \quad-U_{k}^{*}+U_{e e}^{*}=\Delta x^{2} \times\left[\frac{\partial^{2} F}{\partial y^{2}}-v \frac{\partial^{2} F}{\partial x^{2}}\right]_{e} \\
& U_{k}^{*}-4 U_{e}^{*}+3 U_{e e}^{*}=\Delta x^{2} \times\left[\frac{\partial^{2} F}{\partial y^{2}}-v \frac{\partial^{2} F}{\partial x^{2}}\right]_{e e}, \quad U_{k}^{*}=0
\end{aligned}
$$

The terms at the right-hand side of Equations (20a-c) are formulated using the stencil of Equation (16a). Likewise, Equation (8b) is applied at nodes k, n, and nn using Equations (10c) and (15b) as follows

$$
\begin{aligned}
& V_{k}^{*}-4 V_{n}^{*}+3 V_{n n}^{*}=\lambda \Delta x^{2} \times\left[\frac{\partial^{2} F}{\partial x^{2}}-v \frac{\partial^{2} F}{\partial y^{2}}\right]_{k}, \quad V_{k}^{*}-V_{n n}^{*}=\lambda \Delta x^{2} \times\left[\frac{\partial^{2} F}{\partial x^{2}}-v \frac{\partial^{2} F}{\partial y^{2}}\right]_{n} \\
& -3 V_{k}^{*}+4 V_{n}^{*}-V_{n n}^{*}=\lambda \Delta x^{2} \times\left[\frac{\partial^{2} F}{\partial x^{2}}-v \frac{\partial^{2} F}{\partial y^{2}}\right]_{n n}, \quad V_{k}^{*}=0
\end{aligned}
$$

whereby the terms at the right-hand side of Equations (21a-c) are formulated with the stencil of Equation (16b).

Governing equation, moments, and Kirchhoff shear forces at nodes $e, s$ The equations of interior nodes applied. 


\subsubsection{Displacement potential function in the vicinity of beam angles}

The node distribution is the same as that of the ASF-FDA, as shown in Figure 3.

\section{DPF Finite difference approximation at node $k$}

The FDA of the governing equation (Equation (9c)) is expressed with Equation (19), and that of $u(x, y)$ and $v(x, y)$ using Equations (17). The FDAs of the axial/shearing stresses are formulated using Equations (9d-f) and (12a-b) as follows

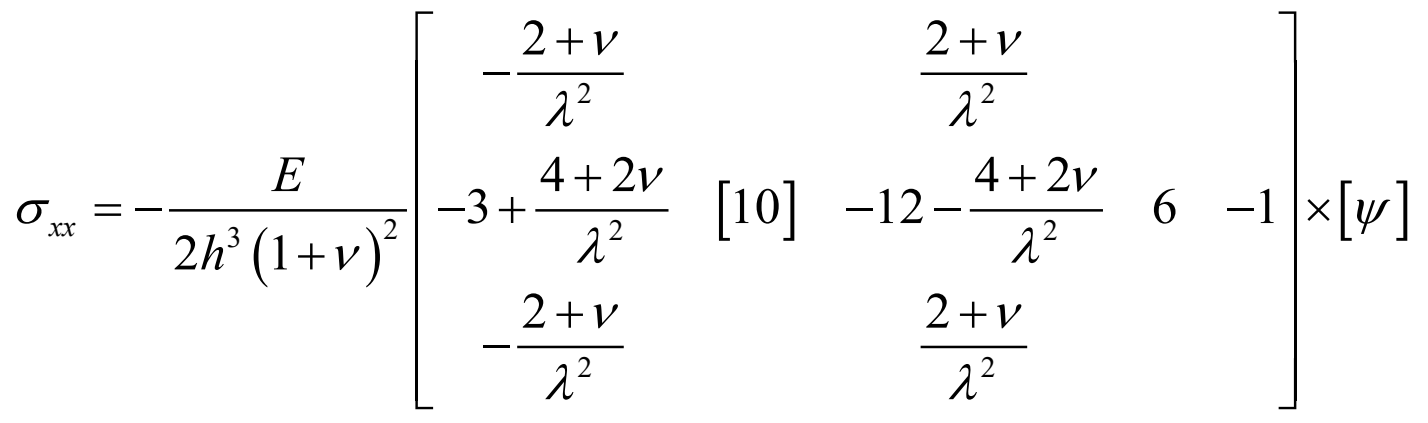

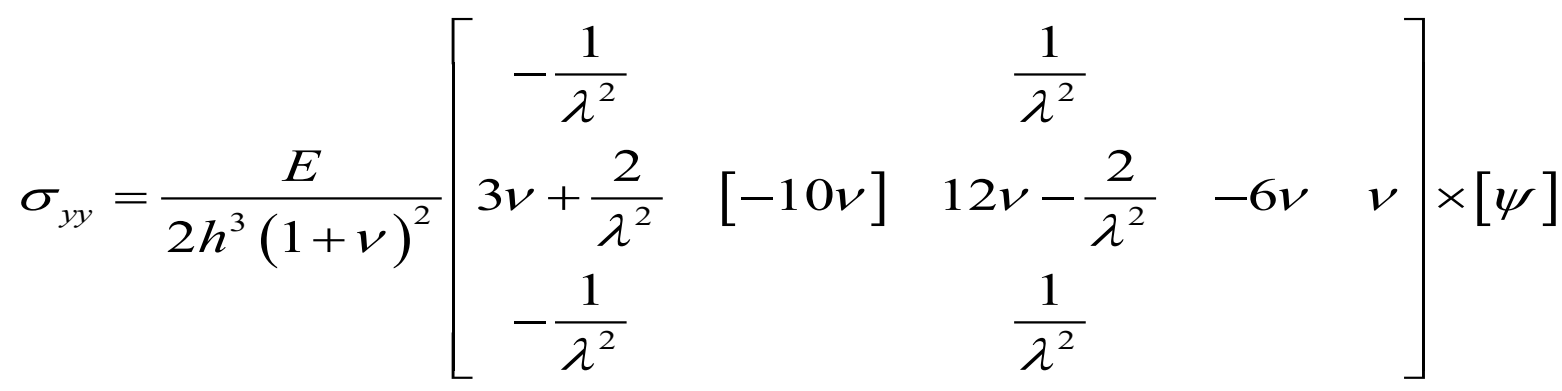

$$
\begin{aligned}
& \tau_{x y}=\frac{E}{2 h^{3}(1+v)^{2}} \times\left[\begin{array}{ccc}
-\frac{v}{\lambda} & \frac{3}{\lambda^{3}}+\frac{2 v}{\lambda} & -\frac{v}{\lambda} \\
& {\left[\frac{-10}{\lambda^{3}}\right]} \\
\frac{v}{\lambda} & \frac{12}{\lambda^{3}}-\frac{2 v}{\lambda} & \frac{v}{\lambda} \\
& -\frac{6}{\lambda^{3}} \\
\frac{1}{\lambda^{3}}
\end{array}\right] \times[\psi]
\end{aligned}
$$

For other angle nodes of the structure, the governing equations are determined similarly, and are presented in Appendix A. The axial/shearing stresses expressed using Equations (12a-b) are displayed in Appendix B.

Governing equation, moments, and Kirchhoff shear forces at nodes e, $s$ The equations of interior nodes applied. 


\subsubsection{ASF-FDA and DPF-FDA at skew edges}

Figure 5 shows a skew edge with regular and additional nodes. The outer normal $\mathbf{n}$ to the skew edge makes an angle $\alpha$ with the $+x$-axis. Two additional nodes are associated to each edge node; therefore, governing equations can be applied at the edge nodes and boundary conditions be satisfied.

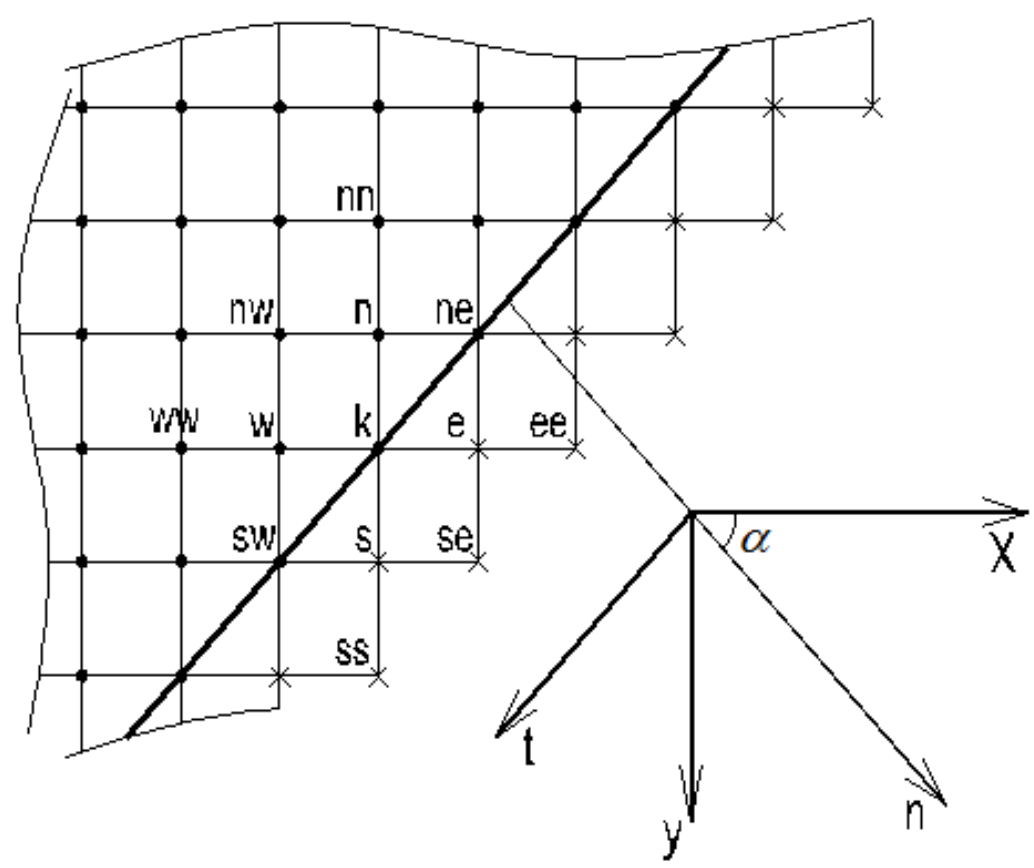

Figure 5 Plate skew edge with regular nodes $(\bullet)$ and additional nodes $(\times)$

The governing equations (Equation (7) or (9c)) can be expressed using Equation (13). Let the axial stresses normal to the skew edge and the shearing stresses tangential to the skew edge be denoted by $\sigma_{\mathrm{n}}$ and $\tau_{\mathrm{t}}$, respectively. The equilibrium equations on an infinitesimal edge element yield the following widely known relationship between $\sigma_{\mathrm{n}} / \tau_{\mathrm{t}}$ and the axial/shearing stresses in Cartesian system

$$
\begin{aligned}
& \sigma_{n}=\sigma_{x x} \cos ^{2} \alpha+\sigma_{y y} \sin ^{2} \alpha+\tau_{x y} \sin 2 \alpha \\
& \tau_{t}=\tau_{x y}\left(\cos ^{2} \alpha-\sin ^{2} \alpha\right)+\left(\sigma_{y y}-\sigma_{x x}\right) \sin \alpha \cos \alpha
\end{aligned}
$$

The axial/shearing stresses in Cartesian system are expressed using Equations (14) for the ASF approach and Equations (18) for the DPF approach. The boundary conditions depend on the displacements and the axial/shearing stresses $\sigma_{\mathrm{n}}$ and $\tau_{t}$; at a free skew edge i.e. they are given by $\sigma_{\mathrm{n}}=0$ and $\tau_{\mathrm{t}}=0$. 


\subsubsection{Finite difference approximations of loadings}

Let us determine the FDA of the distributed load in the case of a varying distributed load per unit area $\mathrm{q}(\mathrm{x})$. The FDA, denoted by $\mathrm{q}_{\mathrm{i}}$, can be taken as the average load around the node of interest and is then expressed as follows:

$$
q_{i}=\frac{1}{\Delta x} \int_{i-1 / 2}^{i+1 / 2} q(x) d x
$$

The load $\mathrm{q}_{\mathrm{i}}$ is used to satisfy the boundary conditions, namely $\sigma_{\mathrm{yy}}=-\mathrm{q}_{\mathrm{i}}$ at the node of interest.

\subsection{Analysis at positions of discontinuity}

Positions of discontinuity are positions of application of concentrated forces, supports, openings, and springs.

\subsubsection{Concentrated force $P$ at node $i$}

The concentrated load $\mathrm{P}$ acting in $\mathrm{y}$-direction can be converted into a load per unit area $\mathrm{q}_{\mathrm{i}}$ at the node of interest

$$
q_{i}=P / \Delta x / d
$$

$d$ being the thickness of the beam. The boundary condition $\sigma_{y y i}=-q_{i}$ is applied at the node of interest.

\subsubsection{Support or spring at node i}

\subsubsection{Airy stress function FDA}

In case of a support, the boundary conditions $(u=0, v=0)$ are satisfied using Equations (20) and (21). In case of a concentrated spring of stiffness $\mathrm{K}_{\mathrm{W}}$ acting in $\mathrm{y}$ - direction i.e, the boundary condition is given by

$$
\sigma_{y y i} \times \Delta x \times d=-K_{W} \times v_{i}
$$

and in case of an elastic Winkler foundation of stiffness $\mathrm{kW}$ in $\mathrm{y}$ - direction i.e, the boundary condition is given by

$$
\sigma_{y y i}=-k_{W} \times v_{i}
$$

The axial stress $\sigma_{\text {yyi }}$ is calculated using Equation (14b), and the displacement $V_{i}$ using Equations (21a-c).

\subsubsection{Displacement potential function FDA}

In case of a support, the boundary conditions $(\mathrm{u}=0, \mathrm{v}=0)$ are satisfied using Equations (17). In case of a concentrated spring or an elastic Winkler foundation Equations (26a-b) applied further, whereby the axial stress $\sigma_{\text {yyi }}$ is calculated using Equation (18b) and the displacement Vi using Equations (17b). 


\section{Stress Analysis of Isotropic Deep Beams Using the Finite Difference Method}

\subsubsection{Local grid refinement}

A local grid refinement can be considered at positions where a concentrated load is applied, or at a concentrated support or spring. Refinement nodes (o) are then introduced around the node of interest k, as represented in Figure 6 for ASF-FDA and DPF-FDA. Furthermore, additional nodes $(\times)$ are introduced and the FDAs and derivatives are obtained using Lagrange interpolating polynomials (Equation (27b)).

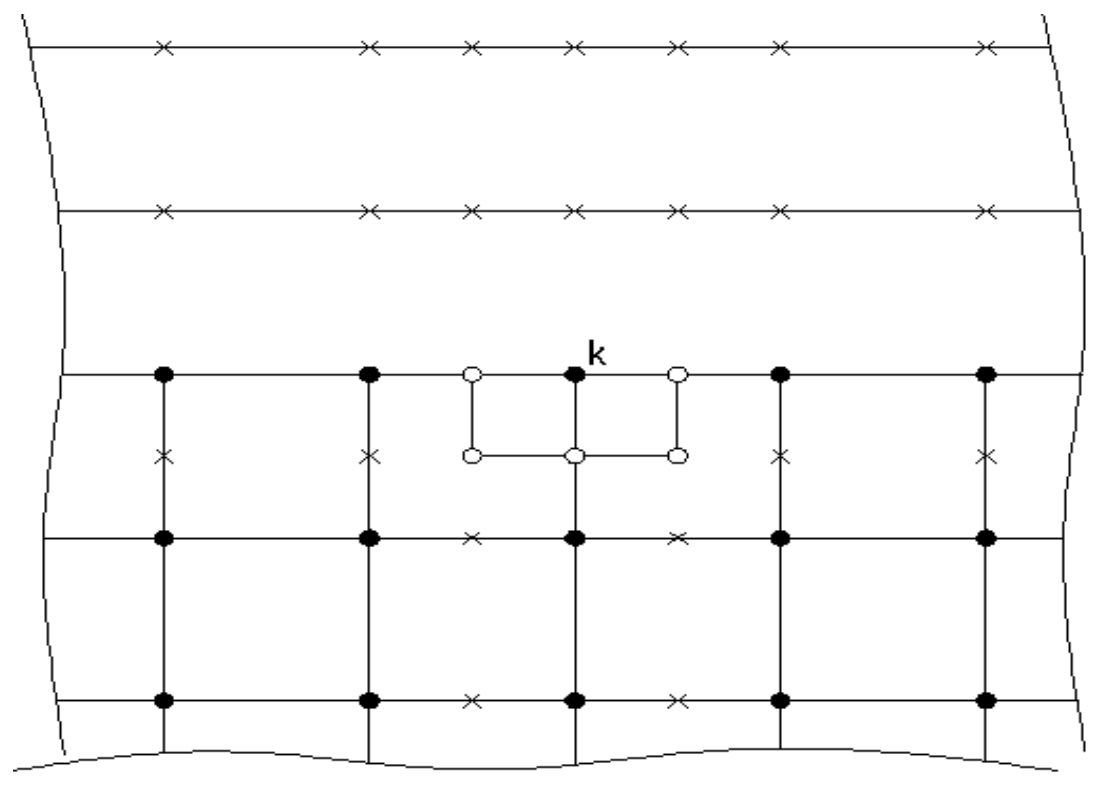

Figure 6 Grid refinement with regular nodes $(\bullet)$, refinement nodes (o), additional nodes $(\times)$

Governing equations (Equations (7) for ASF-FDA and (9c) for DPF-FDA) are applied at regular nodes ( $\bullet$ ) and at refinement nodes $(\mathrm{o})$. However, the Lagrange interpolating polynomials would have to be considered since the grid spacings are not constant in the vicinity of the point of interest.

The Airy stress function and the displacement potential function, represented by $\mathrm{F}(\mathrm{x}, \mathrm{y})$, can be described in $\mathrm{x}$-direction i.e. with values at grid points as follows:

$$
F(x, y)=F_{i-2} \times f_{i-2}(x)+F_{i-1} \times f_{i-1}(x)+F_{i} \times f_{i}(x)+F_{i+1} \times f_{i+1}(x)+F_{i+2} \times f_{i+2}(x),
$$

whereby the shape functions $f_{j}(x)(j=i-2 ; i-1 ; i ; i+1 ; i+2)$ are expressed using the Lagrange interpolating polynomials:

$$
f_{j}(x)=\prod_{\substack{k=i-2 \\ k \neq j}}^{i+2} \frac{x-x_{k}}{x_{j}-x_{k}}
$$




\subsubsection{Deep beams with openings}

A deep beam having an opening is represented in Figure 7 for the ASF-FDA and DPF-FDA, together with regular nodes $(\bullet)$ and additional nodes $(\times)$; the number of additional nodes corresponds to the number of boundary conditions at the node of interest. The unknown at regular nodes and additional nodes is the value of the stress function or the potential function. The additional nodes associated with node $\mathrm{k}$ are denoted by $\mathrm{ka}$ and $\mathrm{kb}$. It is assumed that different nodes may be at the same geometrical position, i.e. nodes $2 \mathrm{a}$ and $8 \mathrm{a}, 3 \mathrm{a}$ and $8 \mathrm{~b}$, ...in Figure 7.

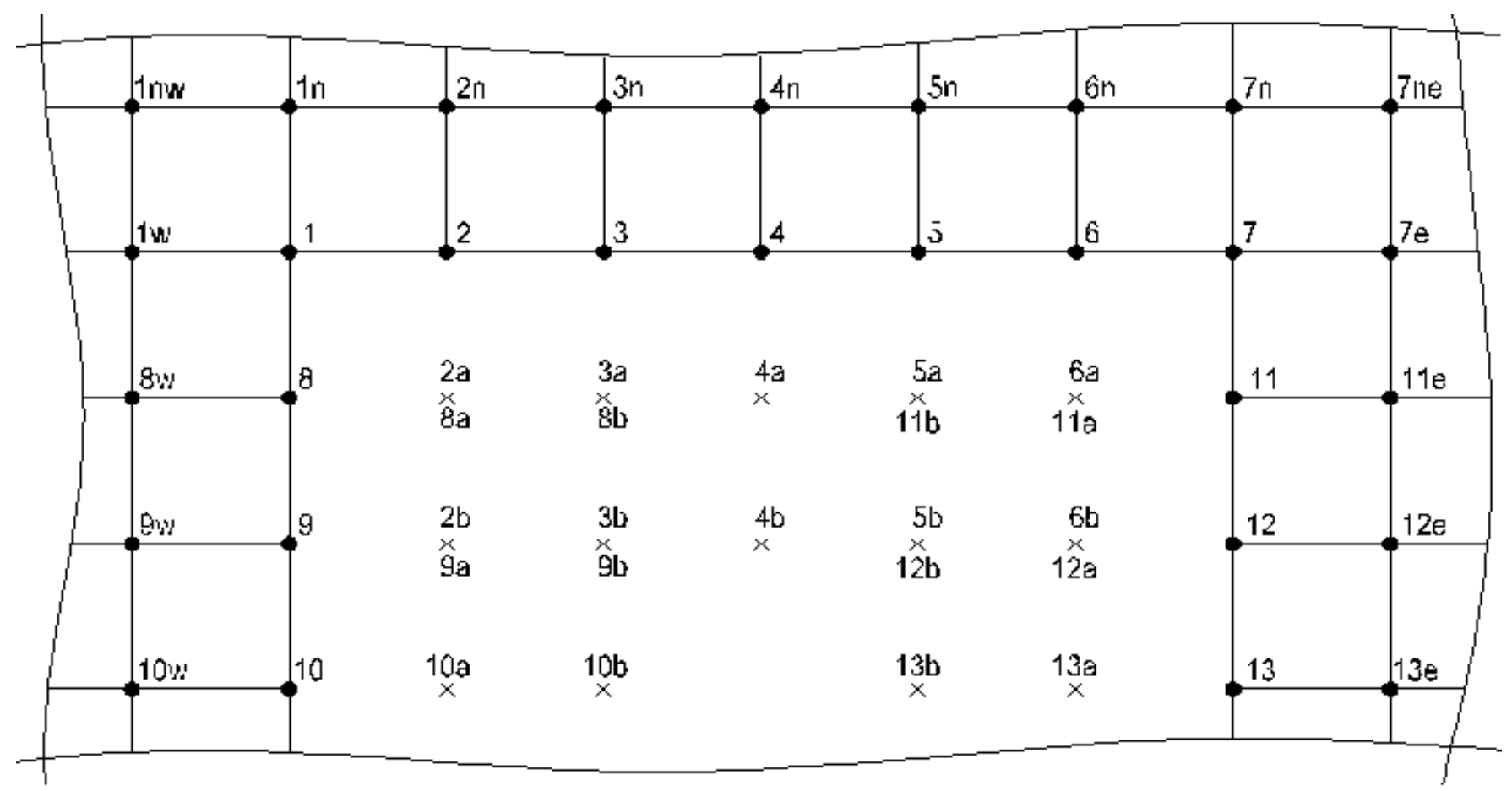

Figure 7 Beam with opening for ASF-FDA and DPF-FDA with regular nodes $(\bullet)$, additional nodes $(\times)$

The function F represents the Airy stress function or the displacement potential function in the following. Particular attention must be taken by the formulation of the governing equations, especially the term $\partial^{4} \mathrm{~F} / \partial \mathrm{x}^{2} \partial \mathrm{y}^{2}$ in the vicinity of angle nodes. The mixed partial derivative $\partial^{4} \mathrm{~F} / \partial \mathrm{x}^{2} \partial \mathrm{y}^{2}$ is expressed using Equation (11a), whereby for angle nodes 1 and 7 the expressions $\left(\mathrm{F}_{2 \mathrm{a}}+\mathrm{F}_{8 \mathrm{a}}\right) / 2$ and $\left(\mathrm{F}_{6 \mathrm{a}}+\mathrm{F}_{11 \mathrm{a}}\right) / 2$ are considered in the stencil; furthermore, at node 2 i.e. it involves the nodes $8,2 \mathrm{a}$, and $3 \mathrm{a}$, and at node 8 it involves the nodes $2,8 \mathrm{a}$, and $9 \mathrm{a}$.

The term $\partial^{4} \mathrm{~F} / \partial \mathrm{x}^{4}$ at nodes $\mathrm{k}(\mathrm{k}=8,9,10,11,12,13 \ldots)$ involves nodes $\mathrm{ka}$ and $\mathrm{kb}$, while the term $\partial^{4} \mathrm{~F} / \partial \mathrm{y}^{4}$ at nodes $\mathrm{i}(\mathrm{i}=2,3,4,5,6)$ involves nodes ia and $\mathrm{ib}$. The boundary conditions are not applied at angle nodes 1 and 7, and they are applied at other nodes using Equations (14) or (18). Especially by the formulation of boundary conditions, the terms $\partial^{2} \mathrm{~F} / \partial \mathrm{x}^{2}$ and $\partial^{3} \mathrm{~F} / \partial \mathrm{x}^{3}$ involve at nodes $\mathrm{k}(\mathrm{k}=8,9,10,11,12,13 \ldots)$ the nodes ka and $\mathrm{kb}$, while $\partial^{2} \mathrm{~F} / \partial \mathrm{y}^{2}$ and $\partial^{3} \mathrm{~F} / \partial \mathrm{y}^{3}$ at nodes $\mathrm{i}(\mathrm{i}=2,3,4,5,6)$ involve nodes ia and $\mathrm{ib}$. 


\subsection{Analysis of isotropic deep beams of variable stiffness}

The axial rigidity D (Equation (5c)) may vary continuously throughout the beam, and is then denoted by D(x,y). Substituting $\mathrm{D}(\mathrm{x}, \mathrm{y})$ into Equations (3a-c) yields the axial/shearing stresses as follows

$$
\begin{aligned}
& \sigma_{x x}=D(x, y) \times\left(\frac{\partial u}{\partial x}+v \frac{\partial v}{\partial y}\right), \quad \sigma_{y y}=D(x, y) \times\left(\frac{\partial v}{\partial y}+v \frac{\partial u}{\partial x}\right), \\
& \tau_{x y}=\frac{1-v}{2} D(x, y) \times\left(\frac{\partial u}{\partial y}+\frac{\partial v}{\partial x}\right)
\end{aligned}
$$

The substitution of Equation (28) into (5a-b) yields the following governing equations

$$
\begin{aligned}
& \frac{\partial}{\partial x}\left[D(x, y) \times\left(\frac{\partial u}{\partial x}+v \frac{\partial v}{\partial y}\right)\right]+\frac{\partial}{\partial y}\left[\frac{1-v}{2} D(x, y) \times\left(\frac{\partial u}{\partial y}+\frac{\partial v}{\partial x}\right)\right]+p_{x}=0 \\
& \frac{\partial}{\partial y}\left[D(x, y) \times\left(\frac{\partial v}{\partial y}+v \frac{\partial u}{\partial x}\right)\right]+\frac{\partial}{\partial x}\left[\frac{1-v}{2} D(x, y) \times\left(\frac{\partial u}{\partial y}+\frac{\partial v}{\partial x}\right)\right]+p_{y}=0
\end{aligned}
$$

Equations (29) are developed and the corresponding FDAs are formulated as described in previous sections. The analysis continues similarly to that of beams of constant stiffness.

\subsection{Analysis of layered beams}

A layered beam presents an abrupt change of material properties apart from a line, as represented in Figure 8.

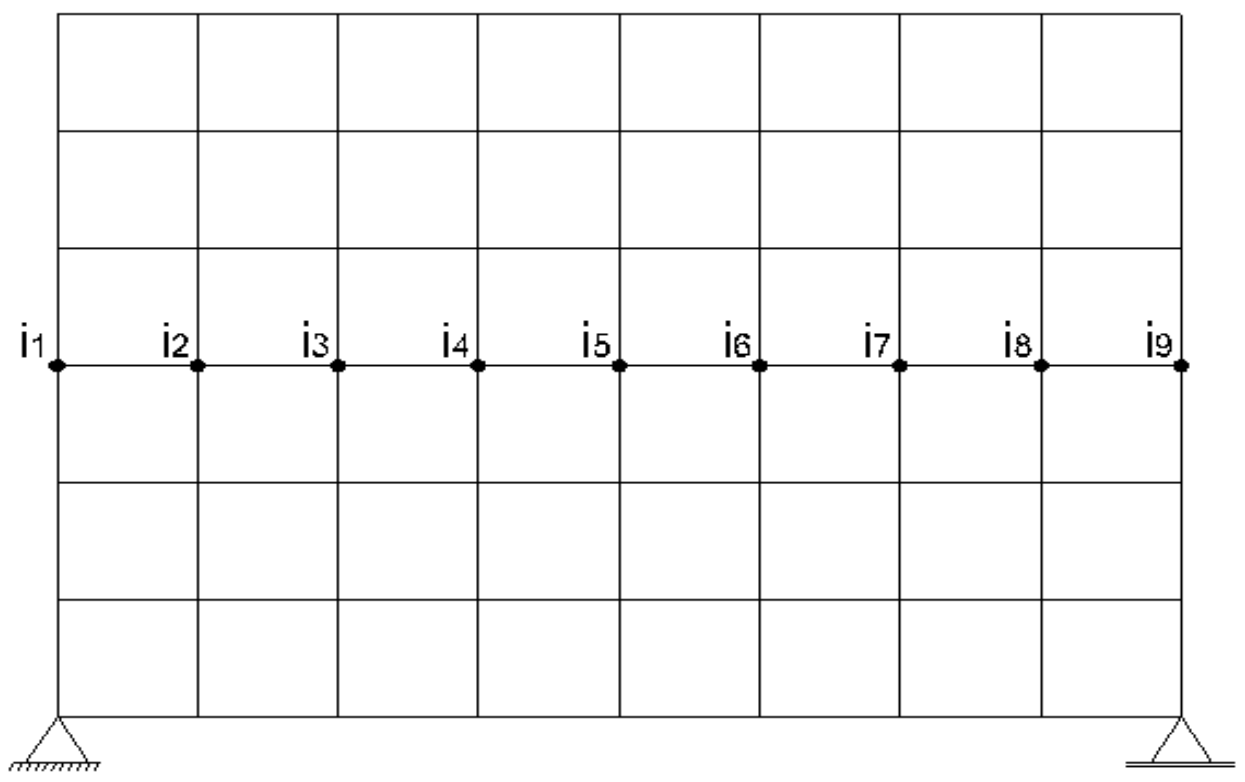

Figure 8 Deep beam with different material properties apart from line $\mathrm{i}$ 
The model developed by Fogang [1] is applied: an opening is realized in the beam along the line i (nodes i1, i2, .., i9) and additional nodes $(\times)$ are introduced in the opening, as represented in Figure 9. The Airy stress function approach and the displacement potential function approach are considered.

\subsubsection{Displacement potential function FDA}

The node distributions are represented in Figure 9.

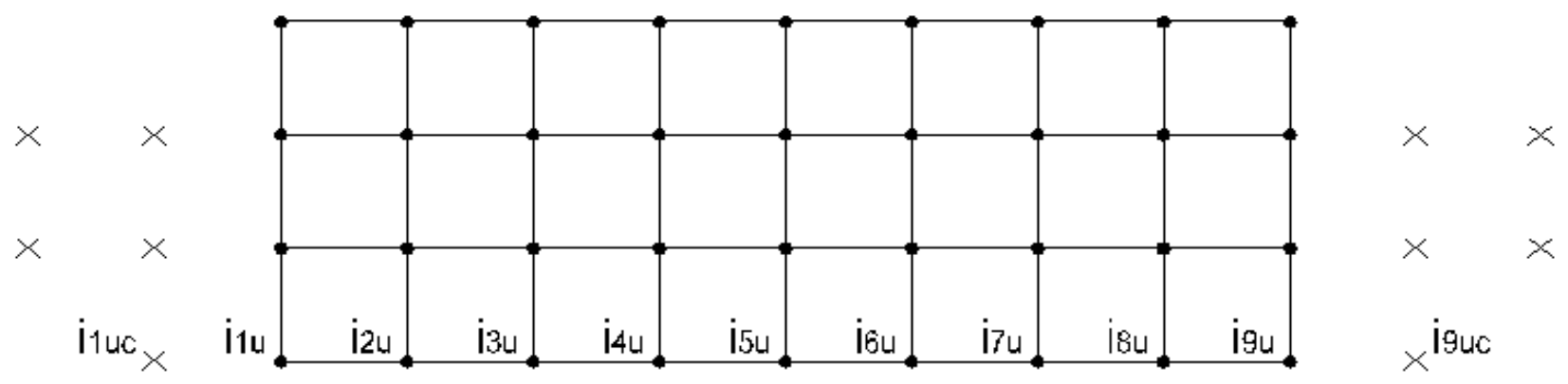

(a)

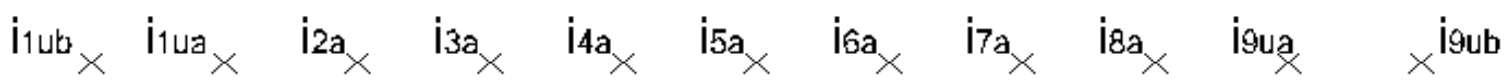

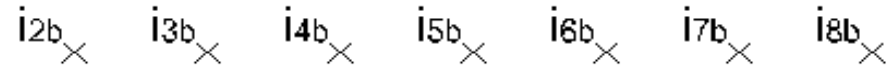

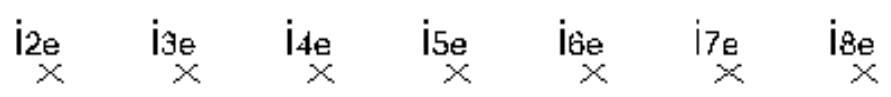

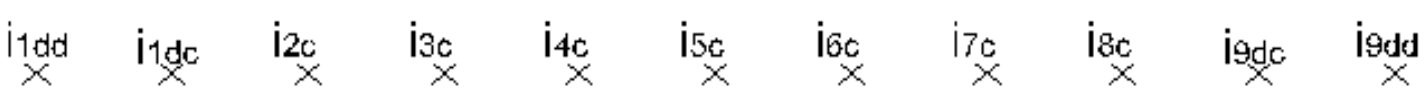

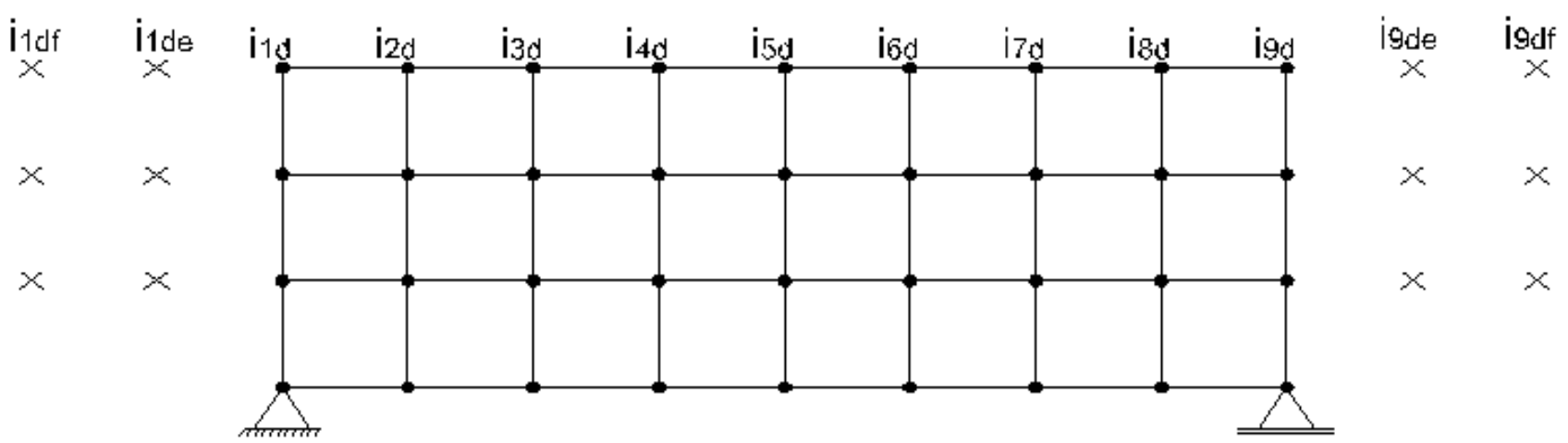

Figure 9 Opening in the beam with regular nodes $(\bullet)$ and additional nodes $(\times)$ : (a) upper part, (b) lower part

The unknown at any node, regular or additional, is the value of the potential function. The number of additional nodes associated to a node in Figure 8 corresponds to the number of continuity equations at the node of interest; therefore, seven additional nodes are introduced at angle nodes il and i9 and four additional nodes at interior nodes. The governing equations, Equation (19) and Appendix A, at angle nodes ild and i9d are modified as follows to take into account the nodes i1df and i9df. 


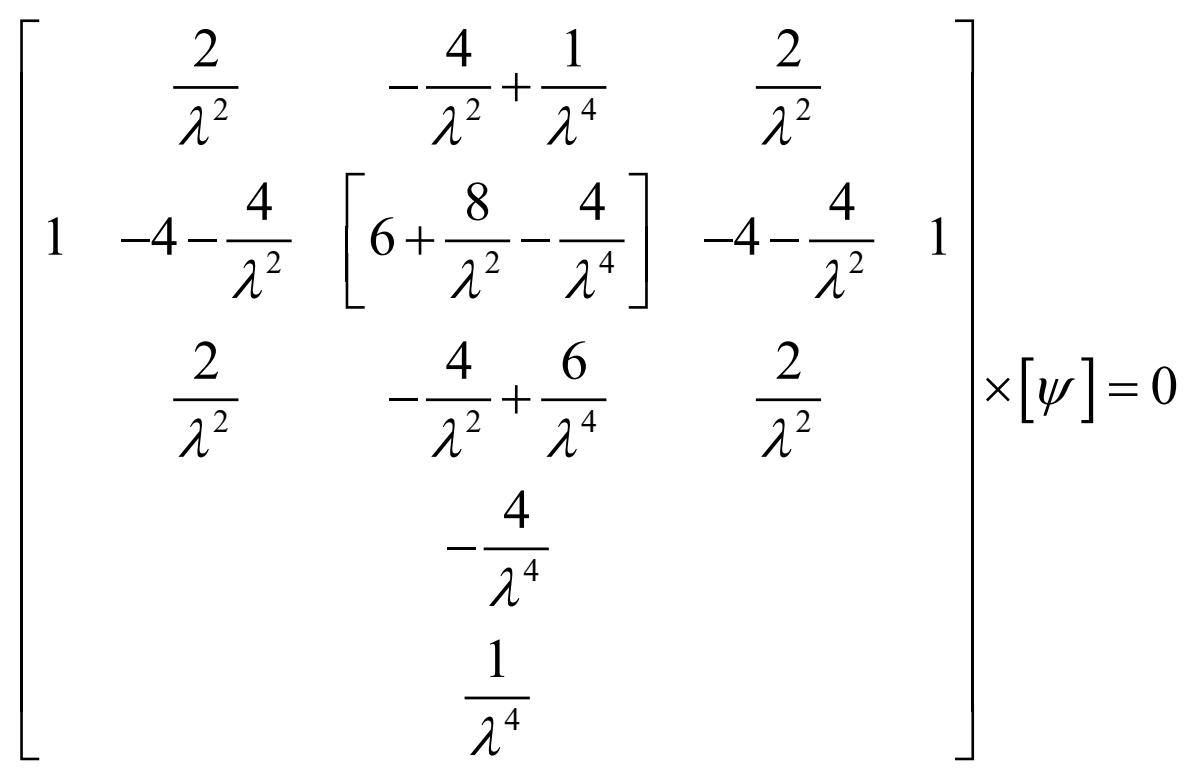

The continuity equations express the continuity of the displacements and the equilibrium of the axial/ shearing stresses. Nodes i1u, i9u, i1d, and i9d: The governing equations are formulated with Equation (19) and Appendix A, and (30a). The axial/ shearing stresses are expressed using Equation (22) and Appendix B, while the displacements are formulated using Equations (17). Alternatively for nodes i1d and i9d the axial stresses can be expressed using Equations (18a-b). The continuity equations between nodes i1u and i1d i.e. are as follows

$$
\begin{array}{ll}
u_{i 1 u}=u_{i 1 d}, & v_{i 1 u}=v_{i 1 d} \\
\sigma_{x x, i 1 u}=0, & \sigma_{x x, i 1 d}=0, \quad \sigma_{y, i 1 u}=\sigma_{y, i 1 d}, \quad \tau_{x y, i 1 u}=0, \quad \tau_{x y, i 1 d}=0
\end{array}
$$

Nodes i2u, i3u, ..., i8u, and i2d, i3d, ..., and i8d: The governing equations (Equation (13)) are applied. The axial/ shearing stresses are expressed using Equation (18), and the displacements using Equations (17). The continuity equations are as follows

$$
u_{i 2 u}=u_{i 2 d}, \quad v_{i 2 u}=v_{i 2 d}, \quad \sigma_{y, i 2 u}=\sigma_{y, i 2 d}, \quad \tau_{x y, i 2 u}=\tau_{x y, i 2 d}
$$

The abrupt change of material properties may occur through a vertical line; in this case a vertical opening is realized and additional nodes are introduced, as represented in Figure 10. 
Stress Analysis of Isotropic Deep Beams Using the Finite Difference Method

$$
\begin{array}{llll}
\times & \times & \times \text { fil } & \\
\times & \times & \times{ }_{\mathrm{fil}} & \times_{\mathrm{flb}}
\end{array}
$$
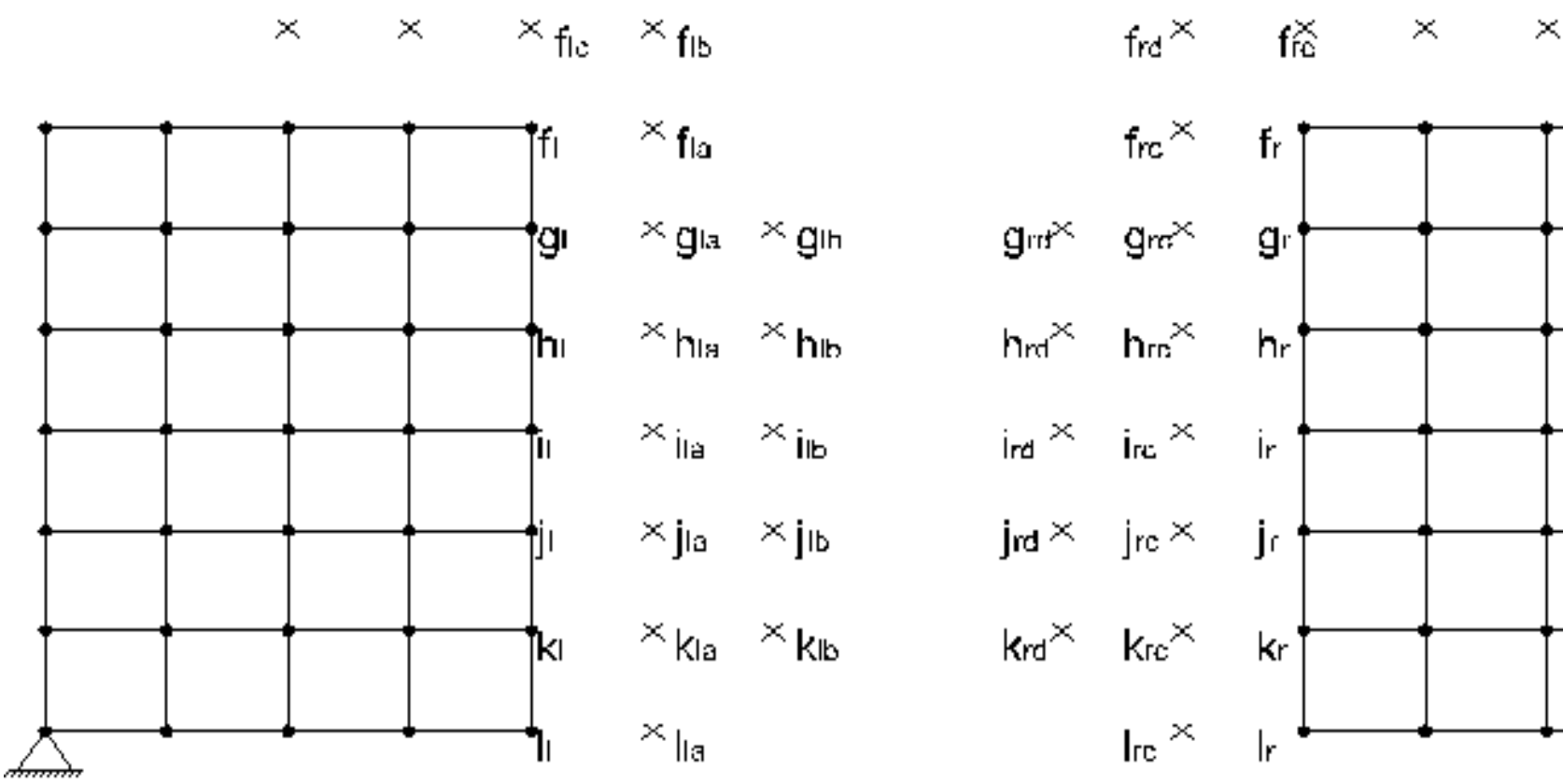

$f_{r c} \times$

$\operatorname{grr} f^{x} \operatorname{grr} x^{x}$

${ }^{\times} h_{\text {h: }}{ }^{\times} h_{\text {his }}$

$h_{r d}{ }^{\times} h_{r r} \times$

$x_{\text {ila }} x_{\text {ilb }}$

$i_{r a} \times i_{i r c}{ }^{x}$

$\times \mathbf{j}_{1 \bullet} \times \times_{j} \mid \bullet$

$\mathrm{jrd}^{\times} \mathrm{j} \mathrm{jr} \times$

${ }^{\times} \mathrm{kla}{ }^{\times} \mathrm{k}_{\mathrm{kb}}$

$\mathrm{krd}^{\times} \mathrm{krc}^{\times}$

$x_{\text {lia }}$

$\operatorname{Irc}{ }^{x}$

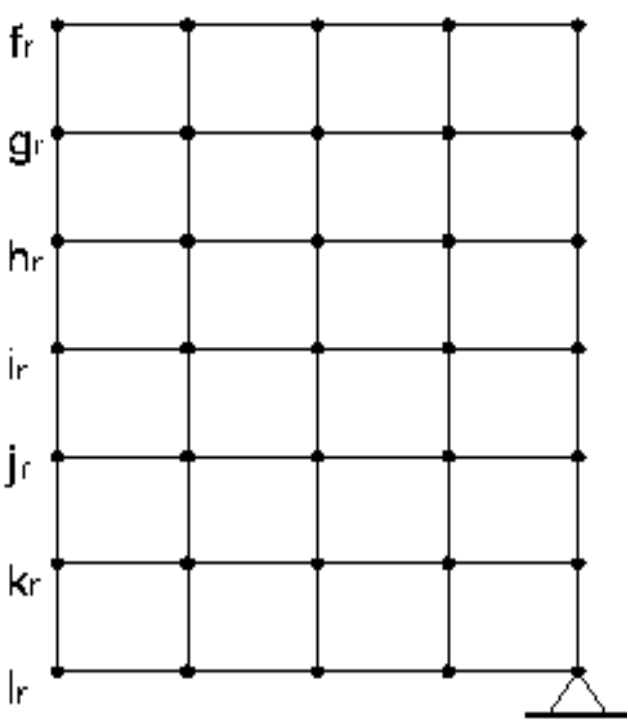

$\times \quad \times \quad x_{I_{\text {I }}} \times \operatorname{lib}_{\text {Ih }}$

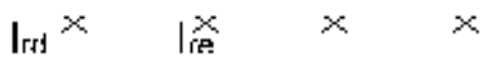

$\times \times x \operatorname{ld}$

Figure 10 Vertical opening in the beam with regular nodes $(\bullet)$ and additional nodes $(\times)$

The governing equations at angle nodes fl and 11 are modified as follows to take into account the nodes fld and $1 \mathrm{ld}$.

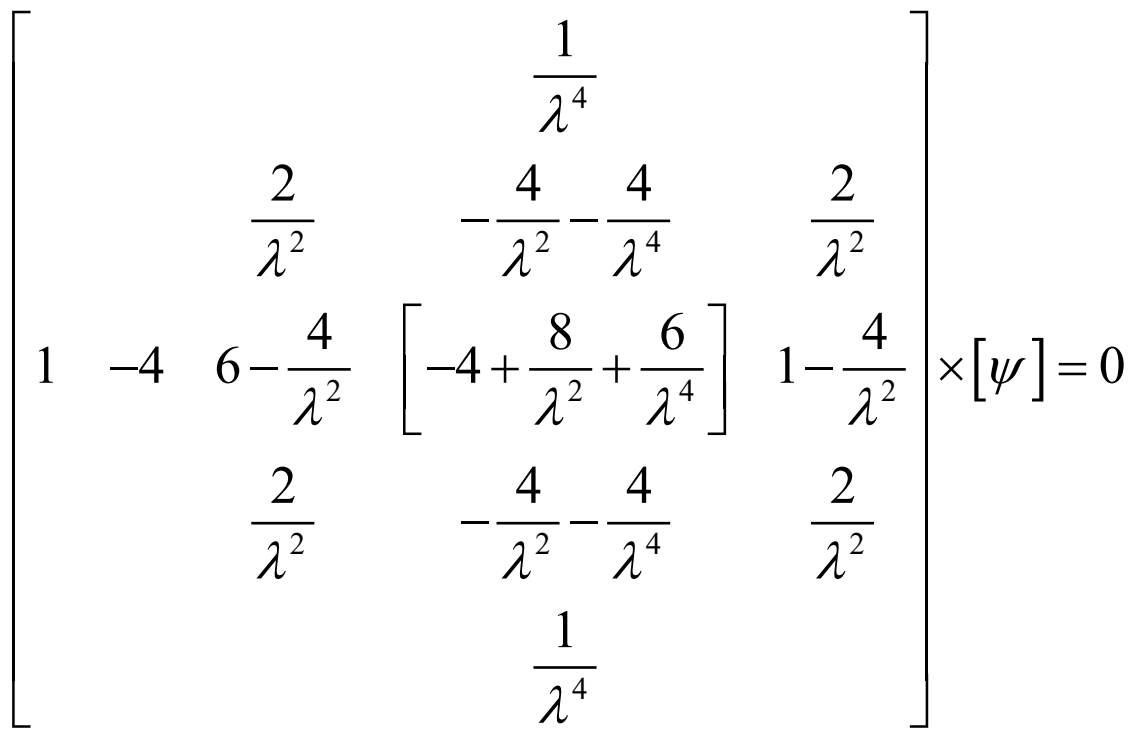


The continuity equations between nodes $\mathrm{il}$ and ir i.e. are as follows

$$
u_{i l}=u_{i r}, \quad v_{i l}=v_{i r}, \quad \sigma_{x x, i l}=\sigma_{x x, i r}, \quad \tau_{x y, i l}=\tau_{x y, i r}
$$

Seven continuity equations between nodes $\mathrm{fl}$ and $\mathrm{fr}$ and between $\mathrm{ll}$ and $\mathrm{lr}$ are applied. No loading is assumed applied at node $\mathrm{f}$. The continuity equations between nodes $\mathrm{fl}$ and fri.e. are as follows

$$
\begin{array}{ll}
u_{f l}=u_{f r}, \quad v_{f l}=v_{f r} \\
\sigma_{y y, f l}=0, \quad \sigma_{y y, f r}=0, \quad \sigma_{x x, f l}=\sigma_{x x, f r}, \quad \tau_{x y, f l}=0, \quad \tau_{x y, f r}=0
\end{array}
$$

\subsubsection{Airy stress function FDA}

The node distribution is the same as that of the displacement potential function approach, and the governing equations are applied similarly. Since the Airy stress function is not directly related to the displacements, the geometric continuity conditions are not easily formulated; for a horizontal separation line they are satisfied as follows:

- u- displacements are introduced at nodes i1u, i2u, .., i9u, and i1d, i2d, .., and i9d. Equations (20a-c) are applied at these nodes

- $\quad \mathrm{v}$ - displacements are introduced at nodes in three lines in upper and lower part of the separation line. Equations (21a-c) are applied at these nodes

- Equations (30b-c) are applied

The analysis is conducted in the same manner for a vertical separation line.

\subsection{Buckling of deep beams}

Fogang [14] derived equations for the buckling analysis of isotropic plates, one of which was as follows

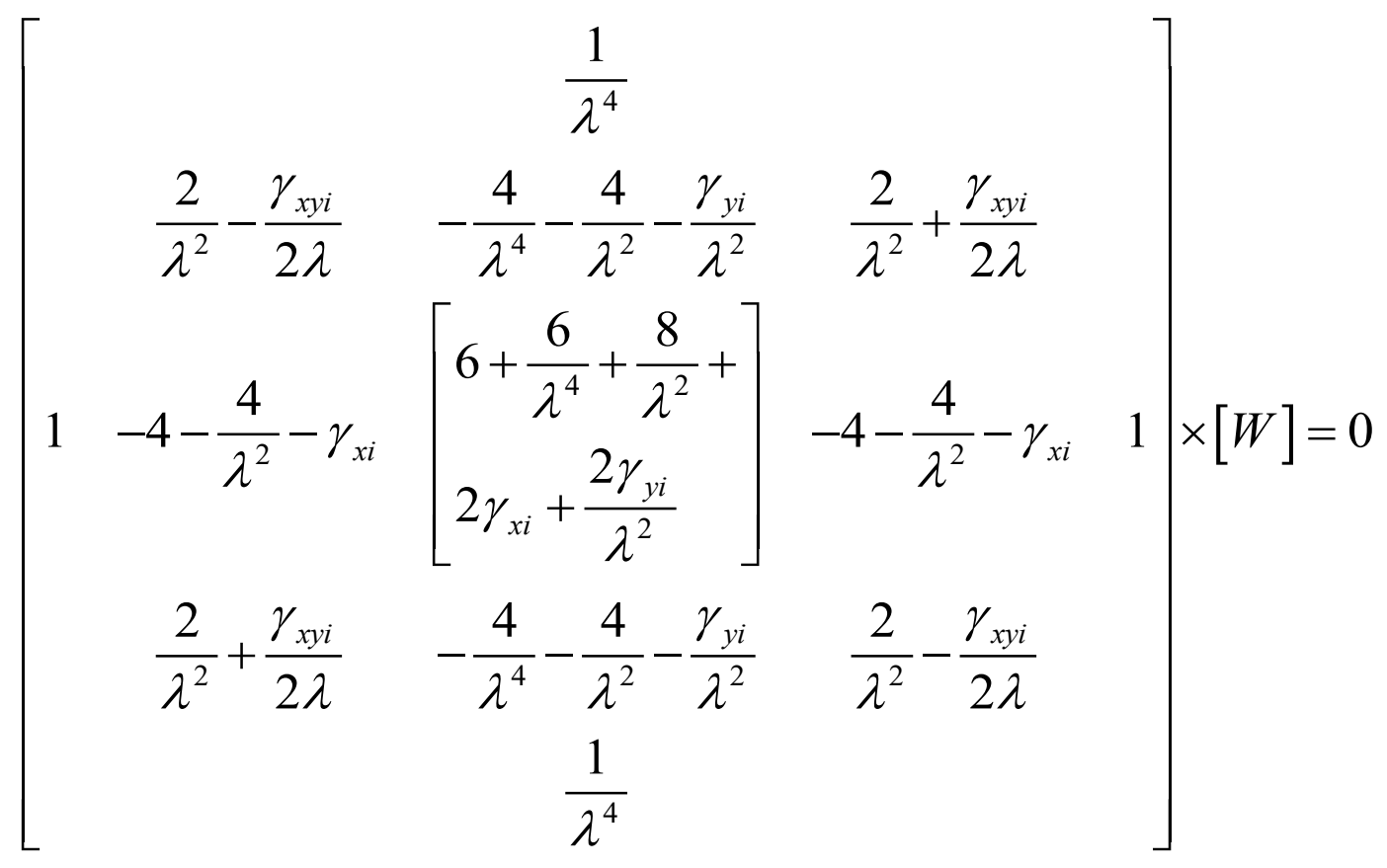


where the grid spacings were $\Delta \mathbf{x}=\mathbf{h}$ and $\Delta \mathbf{y}=\lambda \mathbf{h}$ in $\mathrm{x}$ - and $\mathrm{y}$-direction, respectively, and w was the out-of-plane displacement. The parameters at a node $\mathrm{i}$ were defined as follows

$$
\gamma_{x i}=\frac{n_{x i} h^{2}}{D}, \quad \gamma_{y i}=\frac{n_{y i} h^{2}}{D}, \quad \gamma_{x y i}=\frac{n_{x y i} h^{2}}{D}, \quad D=\frac{E d^{3}}{12\left(1-v^{2}\right)}
$$

where $\mathrm{n}_{\mathrm{xi}}, \mathrm{n}_{\mathrm{yi}}$, and $\mathrm{n}_{\mathrm{xyi}}$ were the axial/shearing forces per unit length at node $\mathrm{i}, \mathrm{D}$ was the flexural rigidity, and $\mathrm{d}$ was the plate thickness. The axial/shearing forces per unit length are related to the stresses of the present study as follows

$$
n_{x i}=d \times \sigma_{x x i}, \quad n_{y i}=d \times \sigma_{y y i}, \quad n_{x y i}=d \times \tau_{x y i}
$$

Therefore, the axial/shearing forces per unit length at any node are determined according to the present study. Thereafter the buckling analysis of the deep beam, in fact a plate buckling analysis, is carried out using Fogang [14].

\section{Results and discussions}

\subsection{Two point supported deep beam and subjected to a distributed load}

A deep beam resting on a fixed and a rolling support and subjected to a uniformly distributed load per unit area $\mathrm{p}=1.0$ $\mathrm{kN} / \mathrm{m}^{2}$, as shown in Figure 11, was analyzed. An $8 \mathrm{x} 8$ grid was considered, such that $\mathrm{a}=10.0 \mathrm{~m}=8 \Delta \mathrm{x}$ and $\mathrm{b}=8 \Delta \mathrm{y}$.

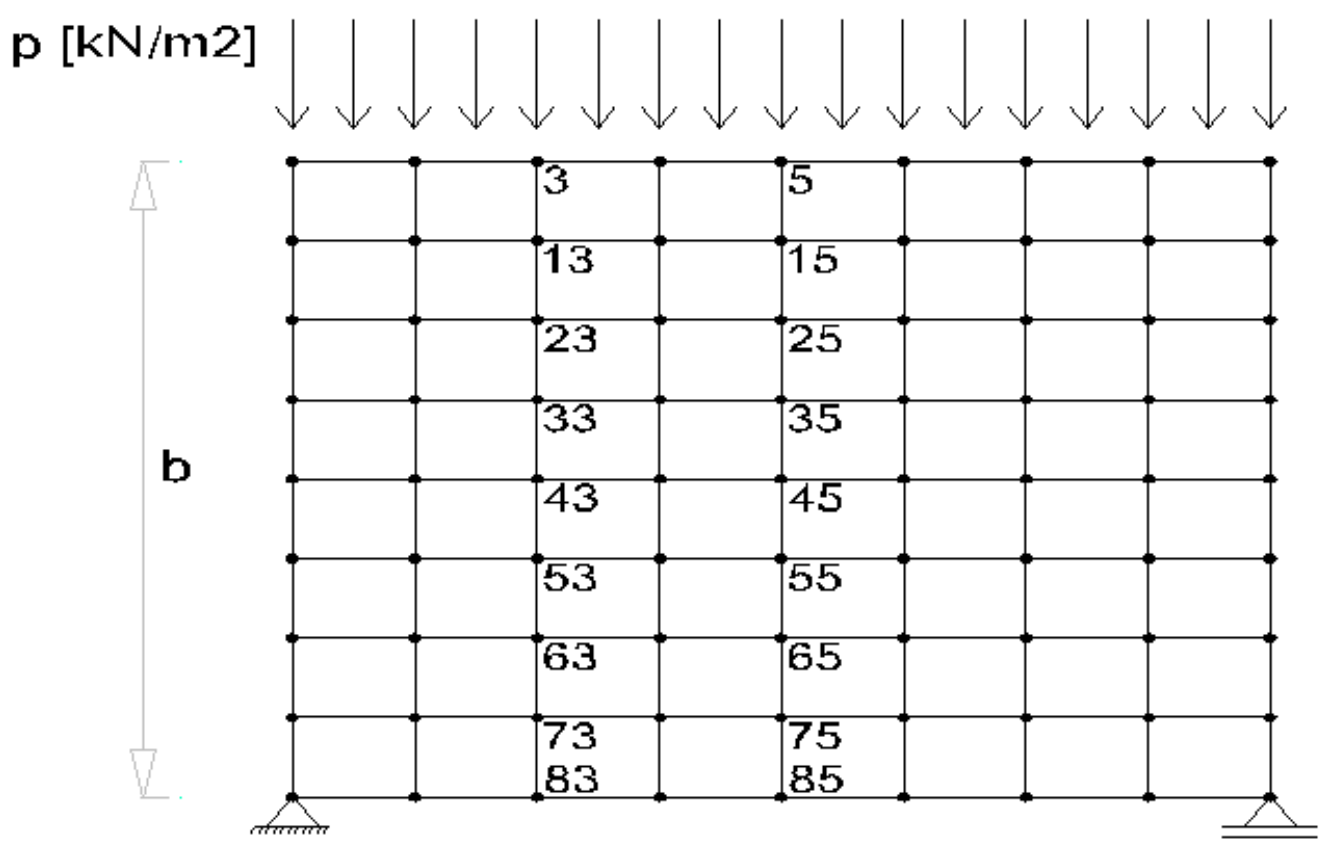

a

Figure 11 Deep beam subjected to a distributed load with regular nodes $(\bullet)$ 
The axial stresses $\sigma_{x x}$ at nodes $5,15 \ldots$, and 85 and the shear stresses $\tau_{x y}$ at nodes $3,13 \ldots$, and 83 are listed in Table 1 and Table 2, respectively, depending on $\lambda=\mathrm{b} / \mathrm{a}=\Delta \mathrm{y} / \Delta \mathrm{x}$. The calculations are conducted using the Airy stress function (ASF) and the displacement potential function (DPF). Results with ASF are also displayed in graphs. Details of the results are presented in the Supplementary file "Two point supported deep beam subjected to a distributed load."

Table 1 Axial stresses $\sigma_{\mathrm{xx}}$ at mid-span of the beam

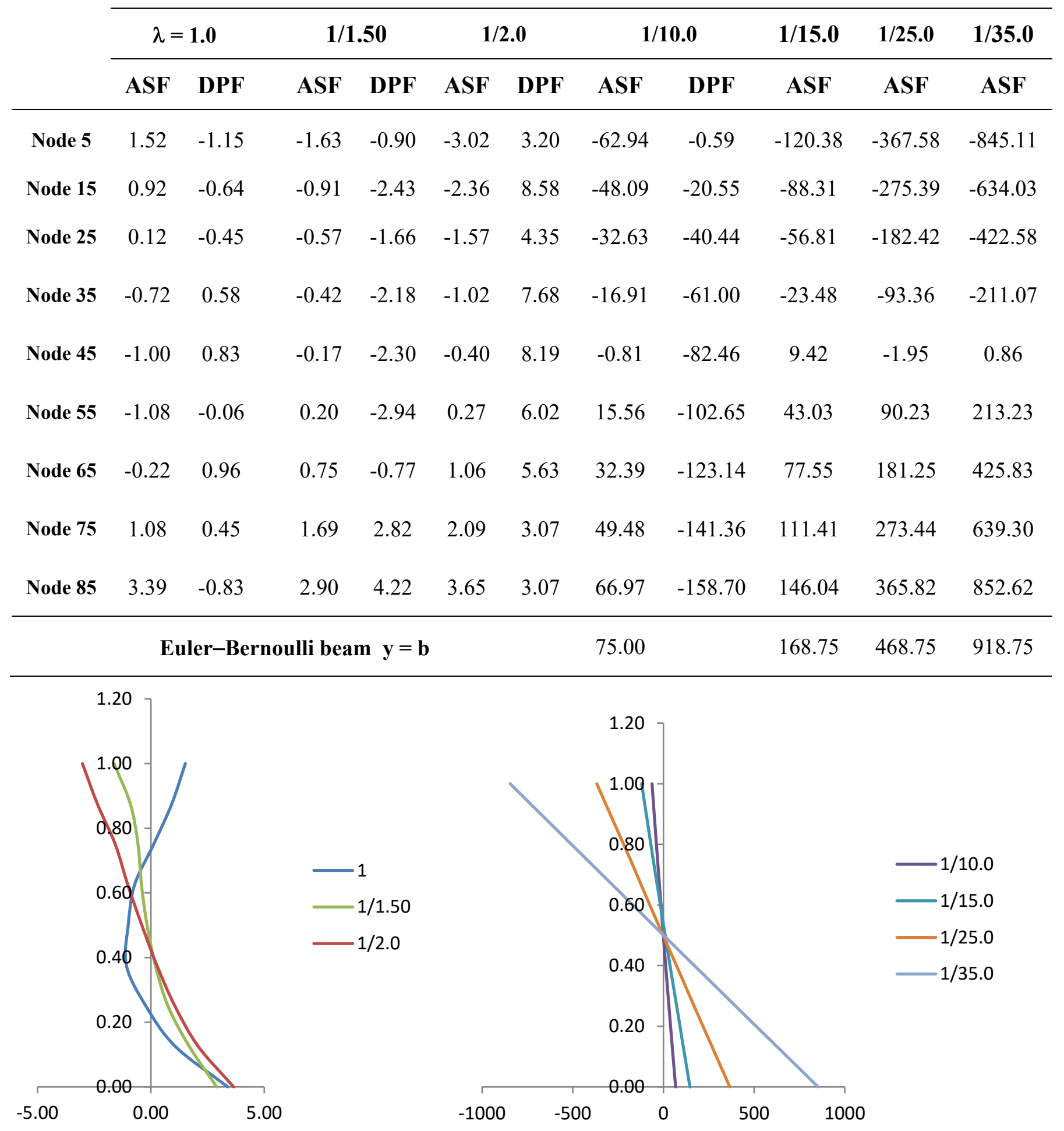


Stress Analysis of Isotropic Deep Beams Using the Finite Difference Method

Table 2 Shearing stresses $\tau_{\mathrm{xy}}$ at position $\mathrm{x}=\mathrm{a} / 4$ of the beam

\begin{tabular}{|c|c|c|c|c|c|c|c|c|c|}
\hline & \multicolumn{2}{|c|}{$\lambda=1.0$} & \multirow{2}{*}{$\begin{array}{c}\mathbf{1} / \mathbf{1 . 5 0} \\
\mathrm{ASF}\end{array}$} & \multirow{2}{*}{$\begin{array}{l}1 / 2.0 \\
\text { ASF }\end{array}$} & \multirow{2}{*}{$\begin{array}{l}\mathbf{1 / 5 . 0} \\
\text { ASF }\end{array}$} & \multirow{2}{*}{$\begin{array}{r}1 / 10.0 \\
\text { ASF }\end{array}$} & \multirow{2}{*}{$\begin{array}{c}\mathbf{1 / 1 5 . 0} \\
\mathrm{ASF}\end{array}$} & \multirow{2}{*}{$\begin{array}{r}1 / 25.0 \\
\text { ASF }\end{array}$} & \multirow{2}{*}{$\begin{array}{r}1 / 35.0 \\
\text { ASF }\end{array}$} \\
\hline & ASF & DPF & & & & & & & \\
\hline Node 3 & -0.05 & & 0.01 & -0.24 & 1.23 & -0.14 & -0.59 & 2.21 & -0.13 \\
\hline Node 13 & -0.36 & & 0.26 & 0.44 & 2.20 & 1.40 & 2.18 & 3.55 & 5.22 \\
\hline Node 23 & -0.55 & & 0.43 & 0.74 & 2.85 & 2.51 & 4.15 & 4.45 & 9.04 \\
\hline Node 33 & -0.23 & & 0.53 & 0.87 & 3.14 & 3.18 & 5.37 & 4.94 & 11.34 \\
\hline Node 43 & 0.31 & & 0.51 & 0.87 & 3.08 & 3.41 & 5.82 & 4.98 & 12.11 \\
\hline Node 53 & 0.67 & & 0.41 & 0.73 & 2.71 & 3.21 & 5.51 & 4.50 & 11.36 \\
\hline Node 63 & 0.76 & & 0.28 & 0.51 & 2.07 & 2.58 & 4.42 & 3.51 & 9.10 \\
\hline Node 73 & 0.45 & & 0.07 & 0.20 & 1.16 & 1.50 & 2.59 & 2.00 & 5.31 \\
\hline Node 83 & 0.03 & & 0.04 & 0.01 & 0.00 & -0.01 & 0.00 & -0.04 & 0.01 \\
\hline
\end{tabular}
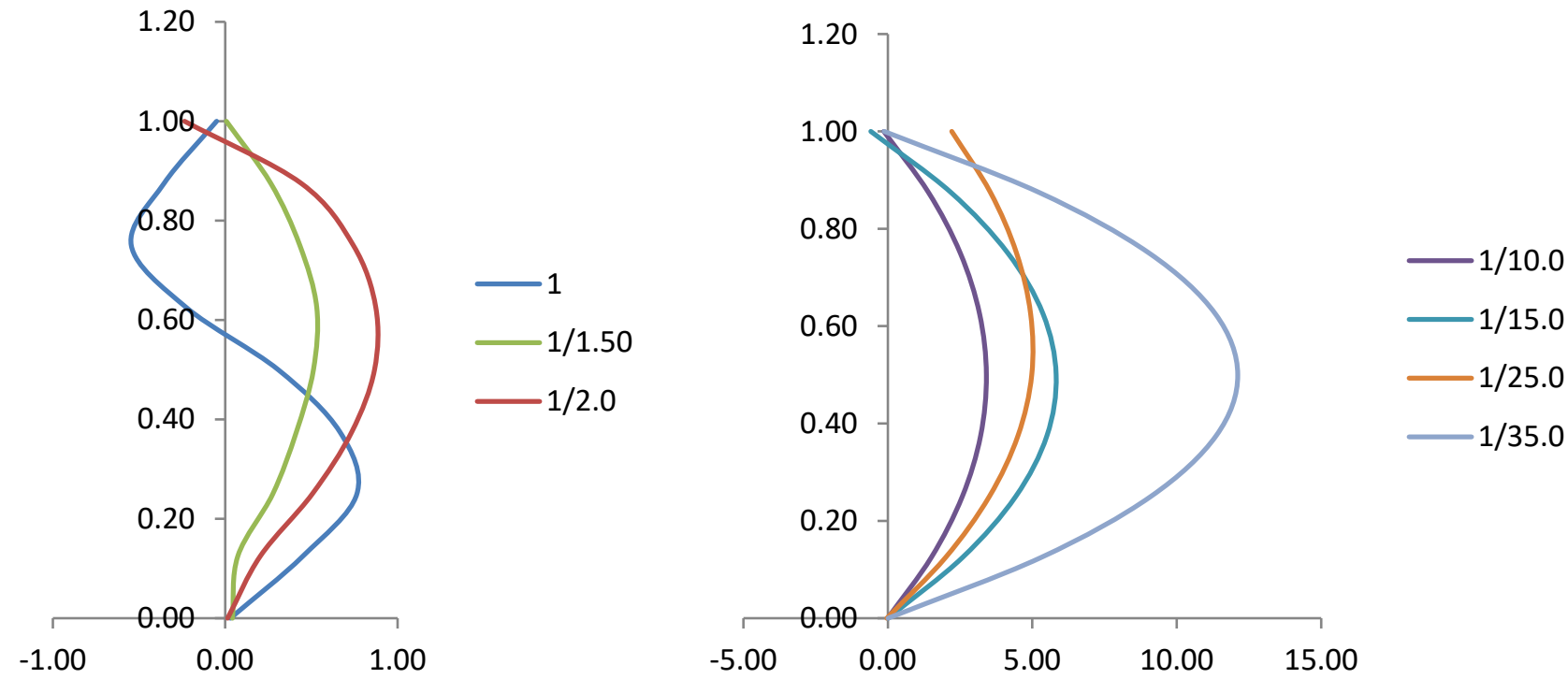

As Table 1 shows, the results obtained using the ASF are reliable; the stresses change sign and for slender beams $(\lambda=$ $1 / 10 ; 1 / 15 ; 1 / 25 ; 1 / 35)$ the stress distribution across the section is linear, what is in agreement with Euler-Bernoulli or Timoshenko beam theory. At the other hand the DPF delivered unreliable results; the stresses do not change sign across the section, therefore $\int \sigma_{\mathrm{xx}} \mathrm{A} \neq 0$ instead of zero as it should be. In Table 2 the shear stresses for slender beams present a parabolic distribution across the section, what is in agreement with Euler-Bernoulli beam theory; however, the top and 
bottom surfaces are not shear free as it should be. In any case, an efficient equation solver is needed for the ASF and DPF approach to have overall reliable results.

\subsection{Two point supported deep beam with an opening and subjected to a distributed load}

A deep beam with an opening resting on a fixed and a rolling support and subjected to a uniform load per unit area $p=1.0$ $\mathrm{kN} / \mathrm{m}^{2}$, as shown in Figure 12, was analyzed. An 8 x 8 grid was considered, such that $\mathrm{a}=8 \Delta \mathrm{x}$ and $\mathrm{b}=8 \Delta \mathrm{y}$. Details of the calculation are presented in the Supplementary file "Two point supported deep beam with opening subjected to a distributed load." Table 3 displays the axial stresses $\sigma_{\mathrm{xx}}$ at mid-span of the beam, at nodes represented in Figure 12.

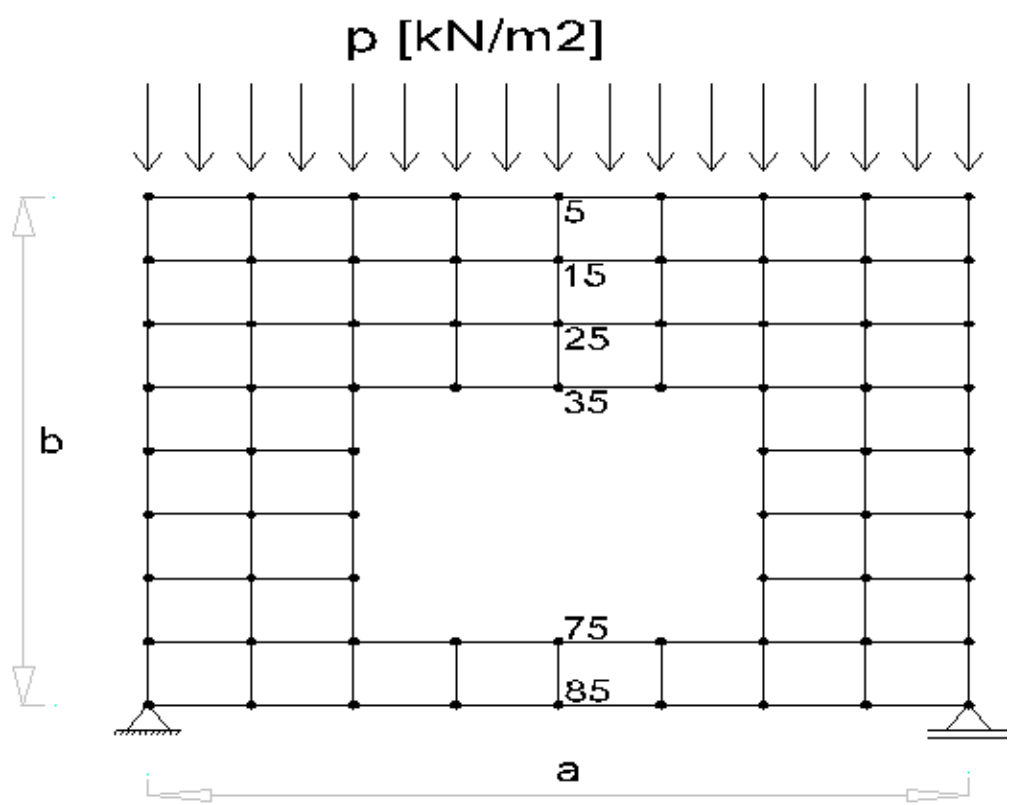

Figure 12 Beam with opening for DPF FDA with regular nodes $(\bullet)$

Table 3 Axial stresses $\sigma_{x x}$ at mid-span of the beam

\begin{tabular}{ccccccccc}
\cline { 2 - 8 } & $\boldsymbol{\lambda}=\mathbf{1 . 0}$ & $\mathbf{1 / 1 . 5 0}$ & $\mathbf{1 / 2 . 0}$ & $\mathbf{1 / 5 . 0}$ & $\mathbf{1 / 1 0 . 0}$ & $\mathbf{1 / 1 5 . 0}$ & $\mathbf{1 / 2 5 . 0}$ & $\mathbf{1 / 3 5 . 0}$ \\
\cline { 2 - 8 } & $\mathbf{A S F}$ & $\mathbf{A S F}$ & $\mathbf{A S F}$ & $\mathbf{A S F}$ & $\mathbf{A S F}$ & $\mathbf{A S F}$ & $\mathbf{A S F}$ & $\mathbf{A S F}$ \\
\hline Node 5 & -1.39 & -3.73 & -3.04 & -23.25 & -93.22 & -210.15 & -584.48 & -1146.01 \\
Node 15 & -0.01 & 0.04 & -0.91 & -5.03 & -21.08 & -47.87 & -133.62 & -262.25 \\
Node 25 & 0.80 & 2.59 & 0.68 & 10.67 & 42.94 & 96.97 & 269.99 & 529.54 \\
Node 35 & -0.14 & -1.16 & 2.50 & 7.96 & 33.07 & 74.88 & 208.63 & 409.24 \\
Node 75 & -0.17 & -0.08 & -0.08 & 1.19 & 4.99 & 11.25 & 31.26 & 61.26 \\
Node 85 & 0.40 & 0.40 & 0.40 & 0.40 & 0.40 & 0.40 & 0.40 & 0.40 \\
\hline
\end{tabular}




\section{Stress Analysis of Isotropic Deep Beams Using the Finite Difference Method}

The results are in agreement with the structural behavior of the deep beam. The slender beam $(\lambda=1 / 10 ; 1 / 15 ; 1 / 25$; $1 / 35$ ) can be regarded as a portal frame with supports connected with a tie. Therefore, the upper part (nodes 5, 15, 25, and 35) performs like a beam with compressive and tensile zone, while the lower part (nodes 75 and 85) acts like a tie.

\section{Conclusion}

The finite difference method based model developed in this paper provided a solution to the stress and stability analyses of deep beams. This model consisted of formulating the differential equations with finite differences and introducing additional nodes outside the beam and at positions of discontinuity (openings, brutal change of stiffness's, non-uniform grid spacing). The introduction of additional nodes permitted to apply the governing equations at the boundaries and to satisfy all of the boundary and continuity conditions. An Airy stress function approach and a displacement potential function approach were considered together with strong formulations of equations (equilibrium, kinematic, and constitutive). By the Airy stress function approach, stresses were formulated in terms of the stress function but geometric boundary conditions were not directly formulated; as result, stresses throughout the structure and displacements in the vicinity of supports were delivered. In the displacement potential function approach, displacements and stresses were formulated in terms of the potential function; so all of the boundary conditions, stress related and geometric, were conveniently expressed. Deep beams of varying stiffness, layered beams, and beams having openings were analyzed with the model. The results obtained using the Airy stress function approach were in agreement with a proper understanding of structural behavior; unfortunately, the displacement potential function approach delivered unsatisfactory results, probably due to the use of an inefficient equation solver.

The following aspects were not addressed in this study but could be analyzed with the model in future research:

$\checkmark \quad$ Vibration analysis of deep beams using the two displacement function approach

$\checkmark \quad$ Beams with anisotropic material behavior such as reinforced concrete beams in high reinforced regions

$\checkmark$ Comparison of the results with those of various beam theories namely Euler-Bernoulli, Timoshenko, and other high-order shear deformation theories

$\checkmark \quad$ Calculation of the shear correction factor for beams with rectangular cross-section

$\checkmark \quad$ Kirchhoff plates with openings

\section{However, some study limitations should be acknowledged}

Large deformation theory

Supplementary Materials: The following files were uploaded during submission:

- "Two point supported deep beam subjected to a distributed load,"

- $\quad$ "Two point supported deep beam with opening subjected to a distributed load." 
Stress Analysis of Isotropic Deep Beams Using the Finite Difference Method

\section{Author Contributions:}

Funding:

\section{Acknowledgments:}

Conflicts of Interest: The author declares no conflict of interest.

\section{Appendix A Airy stress function FDA at nodes e, $s$, and se at angles}

The governing equation (Equation (8)) of the beam at angle nodes E, S, and SE, as shown in Figure 13, is described with the following 13-point stencils

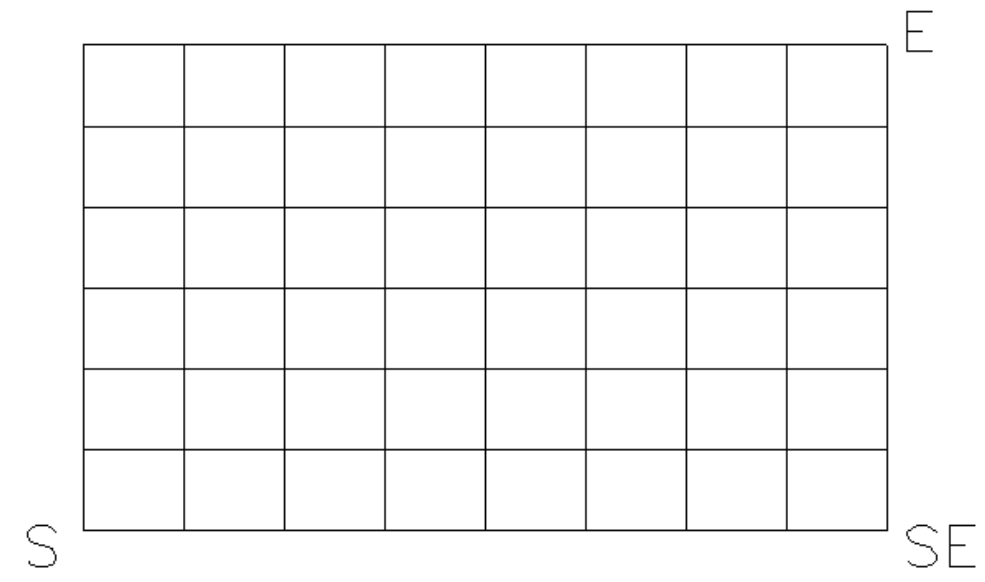

Figure 13 Deep beam with angles E, S, and SE

Node E

$$
\begin{aligned}
& \frac{2}{\lambda^{2}} \quad-\frac{4}{\lambda^{2}}+\frac{1}{\lambda^{4}} \quad \frac{2}{\lambda^{2}} \\
& 1-4 \quad 6-\frac{4}{\lambda^{2}}\left[-4+\frac{8}{\lambda^{2}}-\frac{4}{\lambda^{4}}\right] \quad 1-\frac{4}{\lambda^{2}}
\end{aligned}
$$

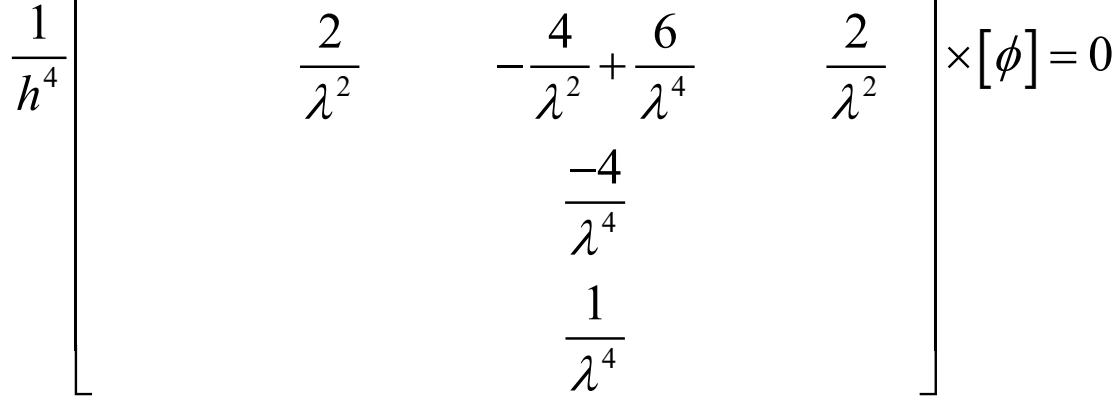


Stress Analysis of Isotropic Deep Beams Using the Finite Difference Method

Node $S$

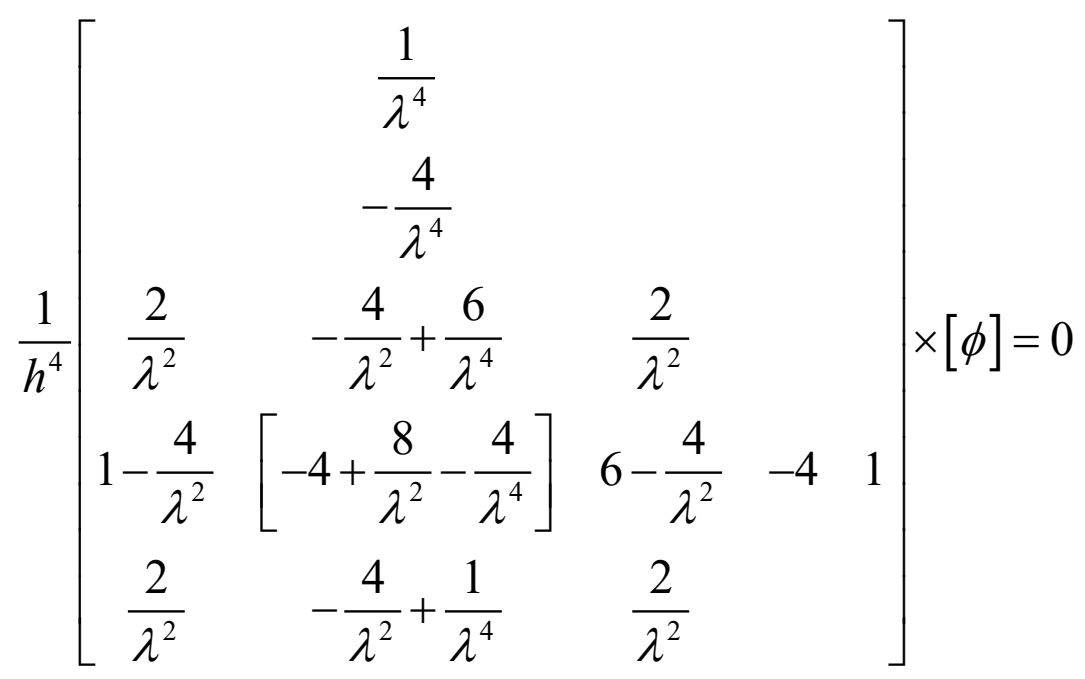

Node SE

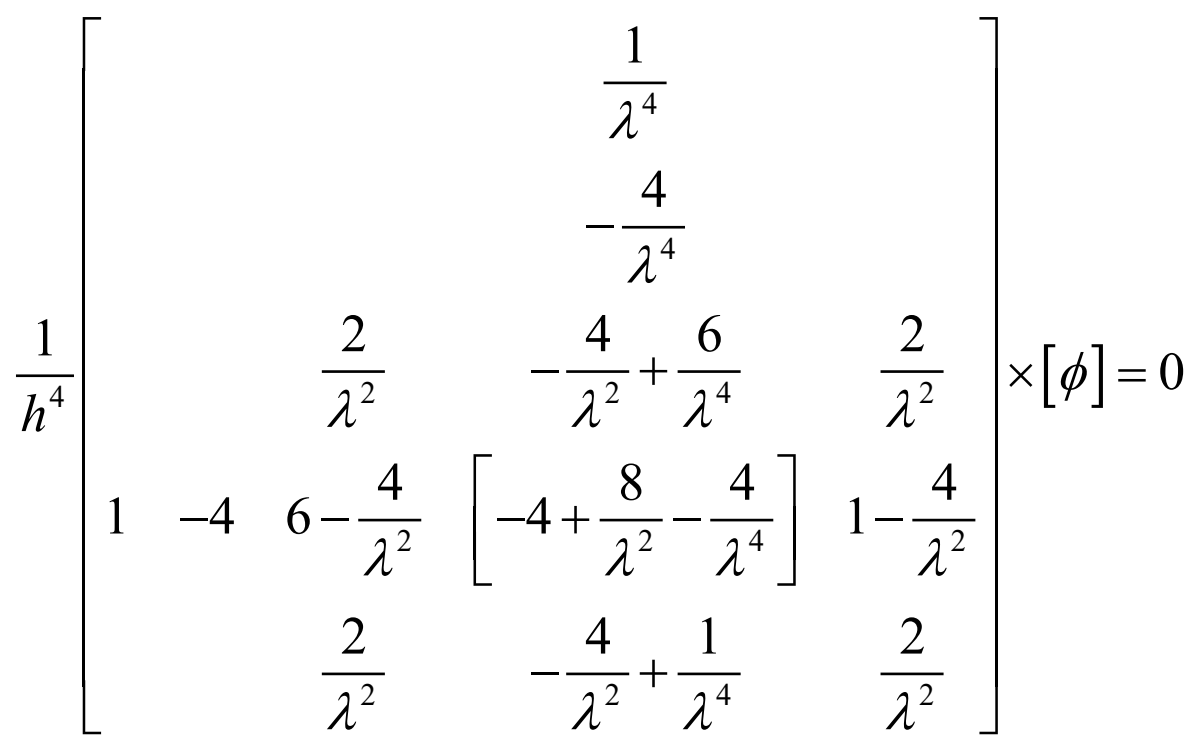

\section{Appendix B \\ Displacement potential function FDA at nodes e, $s$, and se at angles}

The axial/shearing stresses of the beam at angle nodes E, S, and SE, as shown in Figure 13, are formulated with the following 13-point stencils

Node E

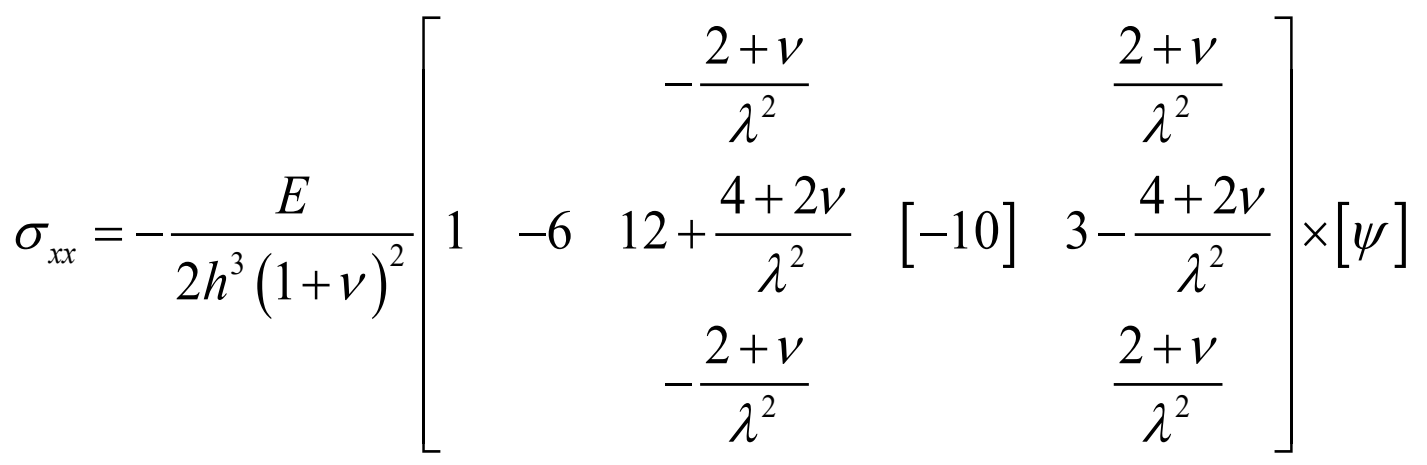




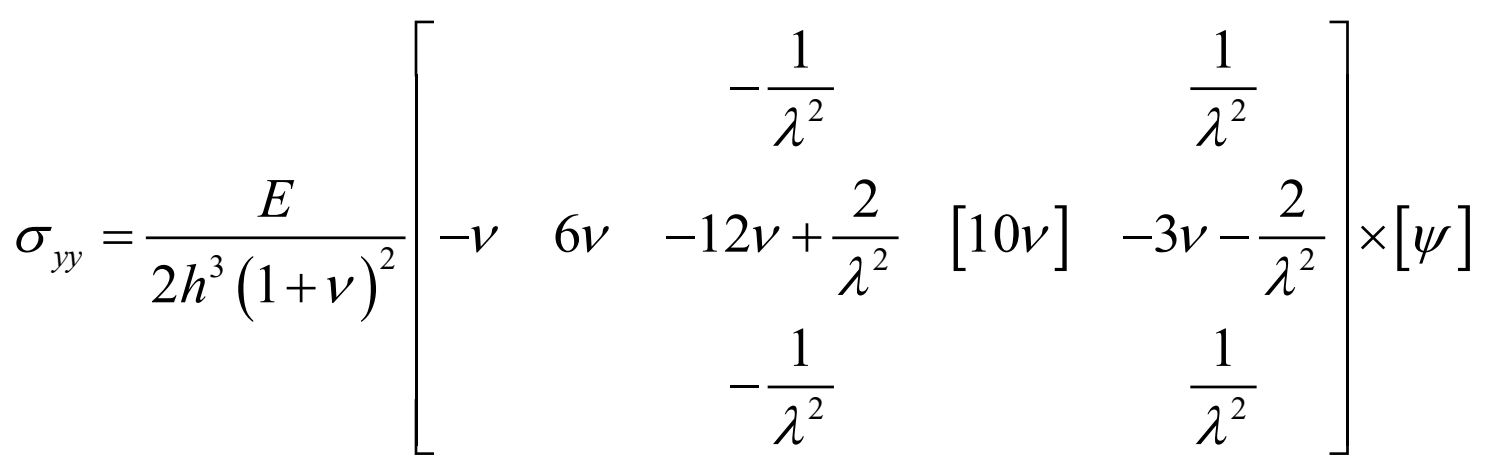

The shearing stress is formulated using Equation (22c).

\section{Node $S$}

The FDAs of the axial stresses are formulated using Equations (22a-b). The shearing stress is as follows

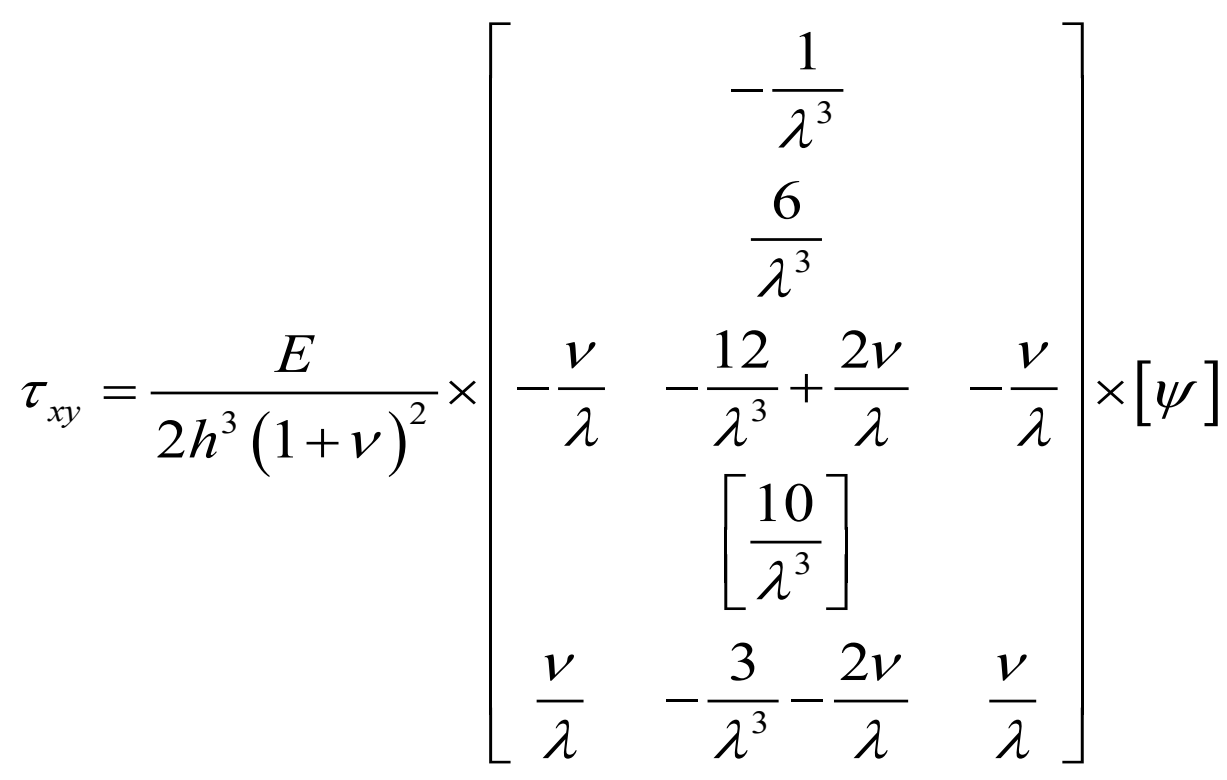

\section{Node SE}

The FDAs of the axial stresses are formulated using Equations (B1) and (B2), and that of the shearing stress using (B3).

\section{References}

[1] Fogang, V. Euler-Bernoulli Beam Theory: First-Order Analysis, Second-Order Analysis, Stability, and Vibration Analysis Using the Finite Difference Method. Preprints 2021, 2021020559

(doi: 10.20944/preprints202102.0559.v3).

[2] Airy, G.B. Brit. Assoc. Advancement Sci. Rept., 1862 


\section{Stress Analysis of Isotropic Deep Beams Using the Finite Difference Method}

[3] Neau, C.Y. 1956. A direct method for determining Airy polynomial stress functions. J. Appl. Mech., 23 (3).

[4] Jayne, B. A., Tang, R. C. Power series stress function for anisotropic and orthotropic beams. Institute of forest products, College of forest Resources, University of Washington.

https://wfs.swst.org/index.php/wfs/article/download/1240/1240/0

[5] Ahmed, S.R., Khan, M.R., Islam, K.M.S., Uddin, Md.W. Investigation of stresses at the fixed end of deep cantilever beams. Computers and Structures 69 (1998) 329-338. Elsevier Science Ltd.

[6] Ismail, E., Issa, M.S., Elbadry, K. Finite element analysis of reinforced concrete deep beam with large opening. Beni-Suef University Journal of Basic and Applied Sciences (2021) 10:25.

https://doi.org/10.1186/s43088-021-00104-z

[7] Vilar, M. M. S., Sartorato, M., Santana, H.B., Leite, M.R. Finite elements numerical solution to deep beams based on layerwise displacement field. Journal of the Brazilian Society of Mechanical Sciences and Engineering (2018) 40:477. https://doi.org/10.1007/s40430-018-1391-2

[8] Sri Harsha, G., Poluraju, P., Khed, V. C. Computation of shear strength equation for shear deformation of reinforced concrete deep beams using finite element method. AIMS Materials Science. 2021, Volume 8, Issue 1: 4261. doi: $\underline{10.3934 / m a t e r s c i .2021004}$

[9] Schlaich, J., Schäfer, K., Jennewein, M. (1987). Toward a consistent design of structural concrete. PCI Journal, Vol. 32, No. 3, pp. 74-150.

[10] Liu, J., Mihaylov, B. Shear strength of RC deep beams with web openings based on two-parameter kinematic theory. 2019. Structural Concrete, Volume 21, Issue 1 p. 349-361. https://doi.org/10.1002/suco.201800356

[11] Silveira, M. V. G., De Souza, R. A. Analysis and design of reinforced concrete deep beams using the stress fields method. Acta Scientiarum. Technology, vol. 39, 05, pp. 587-594, 2017.

\section{DOI: https://doi.org/10.4025/actascitechnol.v39i5.28409}

[12] De Mello, A.F.A., De Souza, R.A. Analysis and Design of Reinforced Concrete Deep Beams by a Manual Approach of Stringer-Panel Method. Latin American Journal of Solids and Structures 13 (2016) 1126-1151. http://dx.doi.org/10.1590/1679-78252623

[13] Säckel. Lecture notes, Höhere Baumechanik. 1993. Hochschule für Verkerhswesen "Friedrich List” Dresden, Sektion Verkehrsbauwesen, WB Ingenieurbau.

[14] Fogang, V. Kirchhoff-Love Plate Theory: First-Order Analysis, Second-Order Analysis, Plate Buckling Analysis, and Vibration Analysis Using the Finite Difference Method. Preprints 2021, 2021110327 (doi: 10.20944/preprints202111.0327.v1). 


\section{Supplementary Files}

This is a list of supplementary files associated with this preprint. Click to download.

- Graphicalabstract.jpg

- Twopointsupporteddeepbeamsubjectedtoadistributedload.xIsx

- Twopointsupporteddeepbeamwithopeningsubjectedtoadistributedload.xlsx 